\title{
Eikonal phase matrix, deflection angle, and time delay in effective field theories of gravity
}

\author{
Manuel Accettulli Huber $\odot,{ }^{*}$ Andreas Brandhuber $\odot,{ }^{\dagger}$ Stefano De Angelis $\odot,{ }^{\dagger}$ and Gabriele Travaglini $\odot^{\S}$ \\ Centre for Research in String Theory, School of Physics and Astronomy, \\ Queen Mary University of London, Mile End Road, London E1 4NS, United Kingdom
}

(Received 15 June 2020; accepted 5 August 2020; published 21 August 2020)

\begin{abstract}
The eikonal approximation is an ideal tool to extract classical observables in gauge theory and gravity directly from scattering amplitudes. Here we consider effective theories of gravity where in addition to the Einstein-Hilbert term we include nonminimal couplings of the type $R^{3}, R^{4}$ and FFR. In particular, we study the scattering of gravitons and photons of frequency $\omega$ off heavy scalars of mass $m$ in the limit $m \gg \omega \gg|\vec{q}|$, where $\vec{q}$ is the momentum transfer. The presence of nonminimal couplings induces helicityflip processes which survive the eikonal limit, thereby promoting the eikonal phase to an eikonal phase matrix. We obtain the latter from the relevant two-to-two helicity amplitudes that we compute up to oneloop order, and confirm that the leading-order terms in $\omega$ exponentiate à la Amati, Ciafaloni and Veneziano. From the eigenvalues of the eikonal phase matrix we then extract two physical observables, to 2PM order: the classical deflection angle and Shapiro time delay/advance. Whenever the classical expectation of helicity conservation of the massless scattered particle is violated, i.e., the eigenvalues of the eikonal matrix are nondegenerate, causality violation due to time advance is a generic possibility for small impact parameter. We show that for graviton scattering in the $R^{4}$ and FFR theories, time advance is circumvented if the couplings of these interactions satisfy certain positivity conditions, while it is unavoidable for graviton scattering in the $R^{3}$ theory and photon scattering in the FFR theory. The scattering processes we consider mimic the deflection of photons and gravitons off spinless heavy objects such as black holes.
\end{abstract}

DOI: $10.1103 /$ PhysRevD.102.046014

\section{INTRODUCTION}

\section{A. Overview}

One of the exciting applications of scattering amplitudes focuses on the computation of classical observables in gauge theory and gravity such as deflection angles and time delay/advance, or effective Hamiltonians describing the dynamics of binary systems. Early results in this direction date back to [1], where it was already noted that loop amplitudes contribute to classical processes, contradicting the erroneous belief of e.g., [2]. The intimate connection between loops and classical physics was sharpened in [3], and had already been applied in [4] to obtain the classical and quantum $\mathcal{O}\left(G^{2}\right)$ corrections to Newton's potential, where $G$ is Newton's constant. In this approach, gravity is

\footnotetext{
*m.accettullihuber@qmul.ac.uk

†a.brandhuber@qmul.ac.uk

*s.deangelis@qmul.ac.uk

§.travaglini@qmul.ac.uk
}

Published by the American Physical Society under the terms of the Creative Commons Attribution 4.0 International license. Further distribution of this work must maintain attribution to the author(s) and the published article's title, journal citation, and DOI. Funded by SCOAP. treated as an effective theory [5], where one can make predictions at low energy despite the nonrenormalisability of the theory. More recently, a systematic approach employing scattering amplitudes in conjunction with unitarity $[6,7]$ was developed to compute classical quantities in gauge theory and gravity. Classical [8] and quantum [8,9] corrections to Newton's potential can be obtained from a two-to-two scattering amplitude of two massive scalars, in particular narrowing down to terms that have discontinuities in the channel corresponding to the momentum transfer $\vec{q}$ of the process [3,5]. An additional simplification stems from the fact that in the unitarity-based calculation the cuts can be kept in four dimensions, as discrepancies with $D$-dimensional results only give rise to analytic terms, at least at one loop. Unitarity was also applied in [10-13] to compute the deflection angle for light or for gravitons passing by a heavy mass, a quantity that has the advantage of being gauge invariant. We also mention some of the efforts leading to the computation of the effective (Newton) potential at second [14,15], third [16-19], fourth [20-32], fifth $[33,34]$ and sixth [35] post-Newtonian order, following the landmark computation at first post-Newtonian order [36]. Comprehensive reviews on this topic from different perspective can be found in [37-40]. In the postMinkowskian framework, which is natural in the context of 
amplitudes, the current state of the art is at 3PM order $[41,42]$, a result which was recently confirmed in $[35,43]$. Note also the effective one-body approach of [44], recently extended to incorporate the first and second postMinkowskian corrections in [45,46], respectively. For other interesting approaches to extract classical observables in general relativity from amplitudes see [47-57].

\section{B. Gravity with higher-derivative couplings}

Much attention has been devoted to the study of effective theories of gravity obtained by adding higher-derivative interactions to the Einstein-Hilbert (EH) action. In particular, efforts have been made in [58,59] to confront such modifications with gravitational wave observations. It was also noted in [58] that for these effects to be measurable by experiments such as LIGO the cutoff of the effective theory must not be much larger than $\mathcal{O}\left(\mathrm{km}^{-1}\right)$. In [60] we initiated a study of the effects that these higher-derivative terms have on the Newtonian potential and deflection angle. In this paper we sharpen this study by rooting it in the eikonal approximation-specifically, applying it to three types of terms, denoted schematically as $R^{3}, R^{4}$ and FFR, for which we compute the corresponding corrections to the deflection angle and time delay/advance. More in detail, the particular action we consider for the graviton, photon and a massive scalar has the form:

$$
\begin{aligned}
S= & \int \mathrm{d}^{4} x \sqrt{-g}\left[-\frac{2}{\kappa^{2}} R-\frac{1}{4} F^{\mu \nu} F_{\mu \nu}\right. \\
& +\frac{1}{2}\left(D_{\mu} \phi\right)\left(D^{\mu} \phi\right)-\frac{1}{2} m^{2} \phi^{2} \\
& \left.-\frac{2}{\kappa^{2}}\left(\frac{\alpha^{\prime 2}}{48} I_{1}+\frac{\alpha^{2}}{24} G_{3}\right)+\frac{2}{\kappa^{2}} \mathcal{L}_{8}-\frac{\alpha_{\gamma}}{8} F^{\mu \nu} F^{\rho \sigma} R_{\mu \nu \rho \sigma}\right],
\end{aligned}
$$

where

$I_{1}:=R_{\mu \nu}^{\alpha \beta} R_{\rho \sigma}^{\mu \nu} R_{\alpha \beta}^{\rho \sigma}, \quad G_{3}:=I_{1}-2 R^{\mu \nu \alpha}{ }_{\beta} R_{\nu \sigma}^{\beta \gamma} R_{\mu \gamma \alpha}^{\sigma}$,

while

$$
\mathcal{L}_{8}=\beta_{1} \mathcal{C}^{2}+\beta_{2} \mathcal{C} \tilde{\mathcal{C}}+\beta_{3} \tilde{\mathcal{C}}^{2},
$$

where

$$
\mathcal{C}:=R_{\mu \nu \rho \sigma} R^{\mu \nu \rho \sigma}, \quad \tilde{\mathcal{C}}:=\frac{1}{2} R_{\mu \nu \alpha \beta} \epsilon_{\gamma \delta}^{\alpha \beta} R^{\gamma \delta \mu \nu} .
$$

A few comments on the various couplings in (1.1) are in order here.

First, there are two types of $R^{3}$ terms, denoted as $I_{1}$ and $G_{3}$ above. Such terms arise naturally in the low-energy effective description of bosonic string theory. Their effects on gravitational scattering of different matter fields have been discussed recently in [60,61]; specifically for the scattering of two massive scalars, both independent structures $I_{1}$ and $G_{3}$ were found to contribute. On the other hand, for the helicity-preserving deflection of massless particles of spin 0,1 and 2, it was shown in [60] that the $G_{3}$ interaction has no effect. Additional interesting features about the $I_{1}$ and $G_{3}$ couplings are that $I_{1}$ is the only coupling that contributes to pure graviton scattering up to four points $[62,63]$ and is the two-loop counterterm in pure gravity [64], while $G_{3}$ is a topological term in six dimensions. In the following we will be concerned with (helicity-preserving and flipping) scattering of massless gravitons in the background produced by a massive scalar, in which case only the $I_{1}$ structure contributes, hence we will refer to it simply as the $R^{3}$ term, since no confusion can arise. Note that in the case of photons there is no $R^{3}$ contribution to the helicity-flipping process.

The second interaction we study is of the type $R^{4}$. In principle there are 26 independent parity-even quartic contractions of the Riemann tensor [65], but only the seven which do not contain the Ricci scalar or tensor survive on shell in arbitrary dimensions, as can also be seen using field redefinitions [66-68]. In four dimensions these reduce to two independent parity-even structures [58,69], plus one parity-odd structure [59], as shown in (1.3). ${ }^{1}$ In agreement with [69] we find that these interactions induce the following four-point graviton amplitudes: those with allequal helicities, and the amplitude with two positive- and two negative-helicity gravitons (the MHV configuration). If $\beta_{2}$ in (1.3) is nonvanishing, then the all-plus and all-minus graviton amplitudes are independent. We also note that a particular contraction of four Riemann tensors appears in type-II superstring theories where it is the first higherderivative curvature correction to the EH theory, and can be determined from four-graviton scattering [71].

The third interaction we consider is an FFR term, where $F$ is the electromagnetic field strength. It is known to arise in string theory as well as from integrating out massive, charged electrons in the case of electrodynamics coupled to gravity, as discussed in [72,73], and considered more recently in $[74,75]$.

We have also introduced in the action a minimally coupled massive scalar to represent a black hole. ${ }^{2}$ Note that in (1.1) we have excluded terms quadratic in the curvatures since from an effective field theory/on-shell point of view they have no effect to any order in four dimensions, as shown recently in [68].

\footnotetext{
${ }^{1}$ A general approach to find a complete, nonredundant operator basis of dimension six and eight for the effective Standard Model including gravity has been given recently in [70] using the Hilbert series method.

${ }^{2}$ In order to describe charged black holes the real scalar in (1.1) should be replaced by an electrically charged complex scalar.
} 


\section{Physical observables from the eikonal phase matrix}

We now come to the computation of the physical observables of interest-these are the classical deflection angle and the time delay/advance [76] experienced by massless gravitons and photons when they scatter off a (possibly charged) massive scalar. A method ideally suited for obtaining classical observables directly from amplitudes, without passing through intermediate, unphysical quantities, is the eikonal [77-82]. In this approach the relevant amplitudes are evaluated in an approximation where the momentum transfer $|\vec{q}|$ is taken to be much smaller than both the mass $m$ of the heavy scalar and the energy $\omega$ of the massless particle, or more precisely taking $m \gg \omega \gg|\vec{q}|$. Crucial for this is a convenient parametrization of spinor helicity variables for the massless particles in the eikonal limit. The amplitudes thus obtained are then transformed to impact parameter space via a twodimensional Fourier transform. In this space the amplitudes are expected to exponentiate into an eikonal phase, from which one can extract directly the classical (and, if desired, quantum) deflection angle and time advance/delay. Recent applications of this method to this type of problem include [83] for the deflection angle of massless scalars up to 2PM, [11] for photons and fermions up to 2PM order, and up to 3PM order in [84-86]. We also note that [11] showed the equivalence of the eikonal method and the formalism based on the computation of an intermediate potential/ Hamiltonian used for instance in $[10-13,60,61]$.

An important point we wish to make is that in our case, because helicity-preserving as well as helicity-violating processes contribute, the eikonal phase is promoted to an eikonal phase matrix in the space of helicities of the external massless particles, with $(+-)$ and $(-+)$ being the diagonal entries associated to no-flip scattering (in a convention where all particles' momenta are outgoing), while $(++)$ and $(--)$ are the off-diagonal entries, with helicity flip. The associated mixing problem has to be resolved in order to obtain the physical quantities of interest. Whenever the two eigenvalues of the eikonal phase matrix are distinct, a possible violation of causality at small impact parameter arises, as noticed already at tree level in [87]. See also [74,75,88-93] for further discussions an resolutions of this issue in UV-complete theories, $[80,87,94,95]$ for earlier appearances of the eikonal operator and [72,73] for related discussions involving helicity flip and no-flip amplitudes.

\section{Summary of the paper}

We now summarize our results. We have computed the graviton deflection angle and time delay/advance for the three interactions $R^{3}, R^{4}$ and FFR, and in addition the photon deflection and time delay induced by the FFR interaction. The single most important qualitative difference with the EH theory is that the propagation and speed of the massless particle acquire a dependence on its polarization in all cases except the graviton propagation in the presence of the FFR interaction. This generically leads to a time advance at small impact parameter $b$. Interestingly, in the case of graviton scattering due to $R^{4}$ and FFR, causality violation can be avoided if the coefficients of the interactions obey certain positivity constraints which, for $R^{4}$, are in precise agreement with those of [96,97]. For the $R^{3}$ interaction our results are fully consistent with the tree-level findings of [87], extending them to one loop. Note that while we used a massive scalar, [87] used a coherent state to set up the background in which the graviton is deflected. Similarly, the FFR interaction induces superluminal propagation of photons.

An important point is the dependence of the eikonal $S$ matrix $S_{\text {eik }}$ on the energy $\omega$ of the scattered massless particle. In the EH theory, $S_{\text {eik }}$ is expected to take the form $S_{\text {eik }}=e^{i\left(\delta_{0}+\delta_{1}+\cdots\right)}$, where the subscript $L$ denotes the loop order of $\delta_{L}$. For the leading eikonal $\delta_{0}$, this was proven for our kinematic setup in [83], and it is generally expected that the $\delta_{L}$ are linear in $\omega$, although we are not aware of an allorder proof. Both for $R^{3}$ and FFR our results are perfectly aligned with this expectation up to $2 \mathrm{PM}$ order, resulting in an $\omega$-independent deflection angle and time advance/delay. A novelty arises for $R^{4}$ where the corresponding eikonal phase (matrix) scales as $\omega^{3}$ with the graviton frequency.

The rest of the paper is organized as follows. In Sec. II we discuss our kinematic set-up and provide explicit expressions for the spinor helicity variables associated to each massless particle in the eikonal limit. We then discuss some general aspects of the eikonal approximation, in particular the extraction of the phases $\delta_{L}$ from the loop scattering amplitudes. We highlight consistency conditions relating amplitudes in impact parameter space at different loop orders and powers of $\omega$ which will then be explicitly checked in all cases considered. We also quote here the relevant formulae to derive the particle deflection angle and time advance/delay.

Section III contains the computations of all tree-level and one-loop amplitudes relevant for our analysis. As a warm up we consider the EH theory, where we re-discuss the graviton deflection computation of [13]; while it is conventionally assumed that in the classical picture the helicity of the scattered massless projectile is unchanged, we show explicitly that this is the case in the eikonal limit: the flipped-helicity amplitudes are nonzero both at tree level and one loop, but are subleading once the eikonal limit is taken, resulting in a diagonal eikonal phase matrix. We then move on to present the relevant four-point two-scalar twograviton amplitudes with and without helicity flip in the case of $R^{3}, R^{4}$ and FFR, as well as the two-scalar twophoton amplitudes for the FFR case, all at tree and one-loop level. Some of these amplitudes have been calculated here for the first time. While at tree level we present exact expressions, at one loop we work in the eikonal 
approximation and we only consider cuts in the $q^{2}$-channel which produce nonanalytic terms arising from the longrange propagation of two massless particles, following the approach initiated in $[4,8,9,98,99]$. Although in the subsequent section we focus only on classical contributions, arising from triangle integral functions with one internal mass, we quote complete answers for the amplitudes up to one loop including box (needed to check the exponentiation of the tree-level phase matrix) and bubble integrals (generating $\mathcal{O}(\hbar)$ corrections to the physical observables).

Section IV is dedicated to the computation of the leading and subleading eikonal matrices $\delta_{0}$ and $\delta_{1}$, from which we will then obtain the $\mathcal{O}(G)$ and $\mathcal{O}\left(G^{2}\right)$ corrections to the deflection angle and time advance/delay for the four cases considered-scattering of gravitons in the presence of $R^{3}$, $R^{4}$ and FFR terms, and scattering of photons induced by the FFR interaction. We also show the case of graviton scattering in EH to set the scene for the more complicated examples discussed later. In all cases we check the exponentiation of the tree-level eikonal phase matrix explicitly, providing important consistency checks of our calculations. Our main results are given in (4.40), (4.47), (4.55), and in (4.41), (4.48), (4.56), for the graviton deflection angle and time advance/delay in the $R^{3}, R^{4}$ and FFR cases, while the photon deflection and time advance/delay in the FFR theory are given in (4.74) and (4.75), respectively.

A few appendixes complete the paper, where we present relevant integrals, the Feynman rules used for some of our new computations, a list of tree amplitudes, and a derivation of the four-point graviton amplitudes in $R^{4}$ only based on little-group and dimensional analysis considerations.

\section{FROM AMPLITUDES TO THE DEFLECTION ANGLE AND TIME DELAY VIA THE EIKONAL}

In this section we first give a precise definition of the eikonal limit providing an explicit parametrization for all the momenta and spinor-helicity variables we need. We then briefly review the connection between amplitudes in the eikonal limit (Fourier-transformed to impact parameter space) and the eikonal phase matrix, the deflection angle and the time delay.

\section{A. Kinematics of the scattering}

We begin by describing the kinematics of the scattering processes we consider. We denote by $p_{1}$ and $p_{2}$ the four-momenta of the incoming and outgoing scalars, respectively, with $m$ being their common mass, while the momenta of the incoming and outgoing massless particles (gravitons or photons) are $p_{4}$ and $p_{3}$. We will work in the center of mass frame, with the following parametrization:

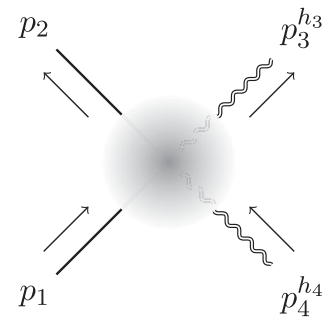

$$
\begin{aligned}
& p_{4}^{\mu}=-\left(E_{4},-\vec{p}+\vec{q} / 2\right), \\
& p_{1}^{\mu}=-\left(E_{1}, \vec{p}-\vec{q} / 2\right), \\
& p_{2}^{\mu}=\left(E_{2}, \vec{p}+\vec{q} / 2\right), \\
& p_{3}^{\mu}=\left(E_{3},-\vec{p}-\vec{q} / 2\right) .
\end{aligned}
$$

In our conventions we take all momenta to be outgoing, hence the minus signs in the expressions of $p_{1}$ and $p_{4}$ since particles 1 and 4 are incoming. We also have

$$
\begin{aligned}
& E_{1}=E_{2}=\sqrt{m^{2}+\vec{p}^{2}+\vec{q}^{2} / 4}, \\
& E_{3}=E_{4}=\sqrt{\vec{p}^{2}+\vec{q}^{2} / 4}:=\omega,
\end{aligned}
$$

where $\vec{p} \cdot \vec{q}=0$ due to momentum conservation. Hence $\vec{q}$ lives in a two-dimensional space orthogonal to $\vec{p}$. In this paper we define the Mandelstam variables as

$s:=\left(p_{1}+p_{2}\right)^{2}=-\vec{q}^{2}, \quad t:=\left(p_{1}+p_{4}\right)^{2}=\left(E_{1}+E_{4}\right)^{2}$,

$u:=\left(p_{1}+p_{3}\right)^{2}$,

with $s+t+u=2 m^{2}$. In this notation the spacelike momentum transfer squared is given by $s$, while $t$ denotes the center of mass energy squared, and $\omega$ is the energy of the scattered massless particle.

In the above parametrization, the kinematic limit we are interested is

$$
m \gg \omega \gg|\vec{q}|,
$$

which implies for the Mandelstam variables

$$
t \simeq m^{2}+2 m \omega, \quad u t-m^{4} \simeq-(2 m \omega)^{2},
$$

and for the energies of the massless particles

$$
E_{3}=E_{4}:=\omega \simeq|\vec{p}|\left(1+\frac{\vec{q}^{2}}{8 \vec{p}^{2}}\right) .
$$

For definiteness we choose $\vec{p}=|\vec{p}| \hat{z}$ with $|\vec{p}| \gg|\vec{q}|$, as implied by (2.4). In this approximation we can write the four-momentum $p_{3}$ of the massless particle in spinor notation as

$$
p_{3}=\left(\begin{array}{cc}
\frac{\vec{q}^{2}}{8|\vec{p}|} & -\frac{\bar{q}}{2} \\
-\frac{q}{2} & 2|\vec{p}|
\end{array}\right),
$$

with $q:=q_{1}+i q_{2}$ and $\bar{q}:=q_{1}-i q_{2}$. One can then find an explicit parametrization for the spinors associated to the null momenta $p_{i}=\lambda_{i} \tilde{\lambda}_{i}, i=3$, 4 , with the result 


$$
\begin{array}{ll}
\lambda_{3}=\sqrt{2|\vec{p}|}\left(\begin{array}{c}
-\frac{\bar{q}}{4|\vec{p}|} \\
1
\end{array}\right), & \tilde{\lambda}_{3}=\sqrt{2|\vec{p}|}\left(\begin{array}{ll}
-\frac{q}{4|\vec{p}|} & 1
\end{array}\right), \\
\lambda_{4}=i \sqrt{2|\vec{p}|}\left(\begin{array}{c}
\frac{\bar{q}}{4|\vec{p}|} \\
1
\end{array}\right), & \tilde{\lambda}_{4}=i \sqrt{2|\vec{p}|}\left(\begin{array}{ll}
\frac{q}{4|\vec{p}|} & 1
\end{array}\right) .
\end{array}
$$

Note the extra factors of $i$ due to the negative energycomponent of $p_{4}$ corresponding to an incoming particle.

\section{B. Eikonal phase, deflection angle and time delay}

In this section we briefly review relevant aspects of the eikonal approximation and the eikonal phase matrix which allows for an efficient extraction of the deflection angle and time delay/advance from scattering amplitudes. This topic was intensively studied in the context of gravity and string theory in the nineties $[81,82]$; for related recent work see also $[55,100,101]$ and references therein.

First, we introduce the amplitude in impact parameter space $\tilde{\mathcal{A}}$. This is defined as a Fourier transform of the amplitude $\mathcal{A}$ with respect to the momentum transfer $\vec{q}$,

$$
\tilde{\mathcal{A}}(\vec{b}):=\frac{1}{4 m \omega} \int \frac{d^{D-2} q}{(2 \pi)^{D-2}} e^{i \vec{q} \cdot \vec{b}} \mathcal{A}(\vec{q}),
$$

where $\vec{b}$ is the impact parameter, and the number of dimensions will eventually be set to $D=4-2 \epsilon$.

In the eikonal approximation the gravitational $S$-matrix can be written in the form $[81,83]$

$$
S_{\text {eik }}=e^{i\left(\delta_{0}+\delta_{1}+\cdots\right)},
$$

where $\delta_{0}$ is the leading eikonal phase, which is $\mathcal{O}(G), \delta_{1}$ the first subleading correction, of $\mathcal{O}\left(G^{2}\right)$, and the dots represent subsubleading contributions. Alternatively, one can write the $S$-matrix in impact parameter space as

$S_{\text {eik }}=1+\tilde{\mathcal{A}}_{\omega}^{(0)}+\tilde{\mathcal{A}}_{\omega^{2}}^{(1)}+\tilde{\mathcal{A}}_{\omega}^{(1)}+\tilde{\mathcal{A}}_{\omega^{3}}^{(2)}+\tilde{\mathcal{A}}_{\omega^{2}}^{(2)}+\tilde{\mathcal{A}}_{\omega}^{(2)}+\cdots$,

where the superscript indicates the loop order $L$ and the subscript the power in the energy $\omega$ of the massless particle. That the maximal power of $\omega$ at a given loop order is $L+1$ is a well-established fact in (super)gravity and we will see below that the $R^{3}$ corrections do not alter this expectation. However, we also find that the $R^{4}$ corrections lead to higher powers of $\omega$ starting at one loop, which is not surprising since higher-derivative corrections worsen the high-energy behavior. In the effective field theory approach we adopt, we are not really interested in high-energy physics (or high-energy completions of the theory)—we use the eikonal approximation as an efficient and elegant tool to extract deflection angles and time delay/advances without passing through the computation of non gauge-invariant intermediate quantities such as effective potentials or Hamiltonians. Nevertheless it would interesting to check if in the $R^{4}$ case unitarity can be restored as well through exponentiation.

Equating (2.10) with (2.11) one gets

$$
\begin{aligned}
& \delta_{0}=-i \tilde{\mathcal{A}}_{\omega}^{(0)}, \\
& \delta_{1}=-i \tilde{\mathcal{A}}_{\omega}^{(1)},
\end{aligned}
$$

as well as the condition

$$
-\frac{\left(\delta_{0}\right)^{2}}{2}=\tilde{\mathcal{A}}_{\omega^{2}}^{(1)}
$$

which implies the consistency condition

$$
\tilde{\mathcal{A}}_{\omega^{2}}^{(1)}=\frac{1}{2}\left(\tilde{\mathcal{A}}_{\omega}^{(0)}\right)^{2} .
$$

Thus, the contribution to the one-loop amplitude that is leading in $\omega, \tilde{\mathcal{A}}_{\omega^{2}}^{(1)}$, does not provide any new information about the $S$-matrix. In general, it is only the term in $\tilde{\mathcal{A}}^{(L)}$ that is linear in $\omega, \tilde{\mathcal{A}}_{\omega}^{(L)}$, that provides new information entering $\delta_{L}$. We also note that (2.12)-(2.15) hold as matrix equations.

Note that a priori these statements are known to hold for EH gravity. The results in this paper show that (2.15) also holds for the higher-derivative interactions discussed here at least up to one loop. Of course the work of [81] on the eikonal limit of string amplitudes gives reason to believe that the exponentiation will work for higher-derivative interactions to all orders.

Finally, the particle deflection angle can be obtained from the eigenvalues $\delta^{(i)}$ of the eikonal phase matrix $\delta$. Using a saddle-point approximation $[11,81,102]$ one finds, for small $\theta$,

$$
\theta^{(i)}=\frac{1}{\omega} \frac{\partial}{\partial b} \delta^{(i)}
$$

where $i$ runs over all eigenvalues of $\delta$ and $b=|\vec{b}|$. For the time delay, we will use instead [103-105]

$$
t^{(i)}=\frac{\partial \delta^{(i)}}{\partial \omega} .
$$

\section{THE RELEVANT SCATTERING AMPLITUDES}

In this section we compute the relevant amplitudes needed to extract the deflection angle and time delay/ advance induced by the various interactions in (1.1). At tree level we will present exact expressions; at one loop we only need to compute the part of the amplitude with a 


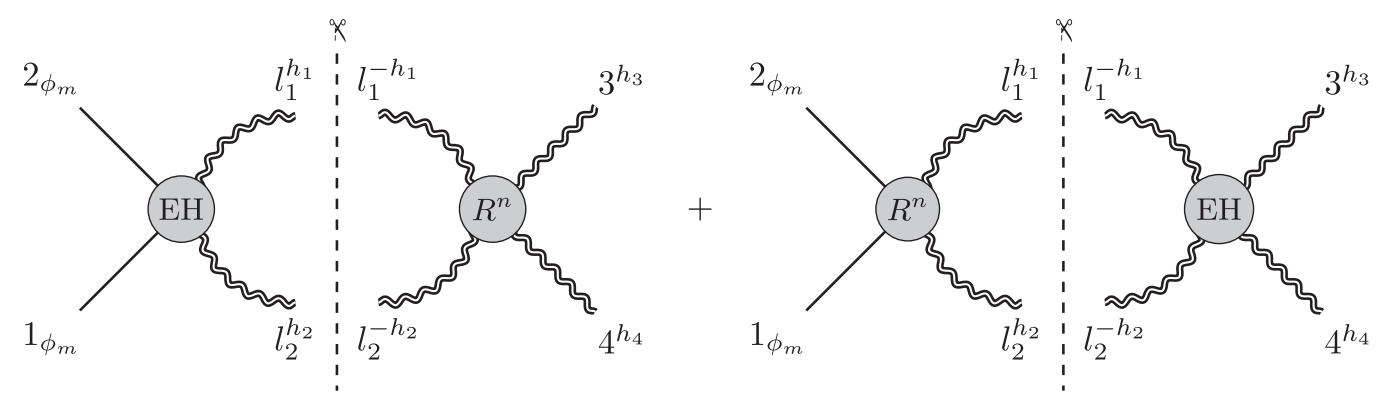

FIG. 1. The two-particle cut diagrams for the $R^{n}$ interaction in the $s=-\vec{q}^{2}$-channel. In our conventions external momenta are all outgoing and internal loop momenta flow from left to right in the diagram.

discontinuity in the $s$-channel ${ }^{3}$ and we will write the relevant expressions after expanding them in the eikonal approximation (2.4) - this will be denoted in the following by the $\simeq$ symbol. A direct extraction of the classical part of the deflection angle and time delay can be performed using triple cuts, and in an even more refined way using the holomorphic classical limit [106]. We chose instead to compute the one-loop amplitudes through two-particle cuts, which also determine the quantum part of the amplitude. The latter, despite not being used in the present paper, becomes essential when considering the exponentiation in the eikonal limit at higher orders [101].

We will begin our discussion with the simple case of $\mathrm{EH}$ gravity, quoting from [13] the relevant two-scalar twograviton amplitude without helicity flip. We also compute the amplitude with helicity flip, and show that it does not contribute in the eikonal approximation, as correctly assumed in previous treatments. We will then move on to compute the relevant tree and one-loop amplitudes that are necessary in order to compute the corrections induced by the $R^{3}, R^{4}$ and FFR terms in (1.1).
The two-particle cut diagrams relevant for the $R^{3}$ and $R^{4}$ cases are shown in Fig. 1. The corrections induced by the FFR interaction need a separate analysis and we show the corresponding diagrams in Figs. 2 and 3. For the case of the $R^{n}$ interaction both internal and external particles are gravitons, while in the case of FFR we either have external gravitons and internal photons, or viceversa.

A comment is in order here. Focusing on the cuts relevant for $R^{n}$ depicted in Fig. 1, the case $h_{3}=h_{4}$ corresponds to the massless particle flipping helicity upon interacting with the scalar, whereas $h_{3}=-h_{4}$ corresponds to the helicity-preserving process, since in our conventions all external particles are outgoing. A simple way to take into account particle statistics is to sum over all values of the internal helicities $h_{1}$ and $h_{2}$ and divide the result by $2{ }^{4}$

\section{A. Four-point scalar/graviton scattering in EH gravity}

The relevant tree-level amplitudes in the EH case are the two-scalar/two-graviton amplitudes in the two helicity configurations for the gravitons ${ }^{5}$ :

$$
\begin{aligned}
& \mathcal{A}_{\mathrm{EH}}^{(0)}\left(1^{\phi}, 2^{\phi}, 3^{--}, 4^{++}\right)=-\left(\frac{\kappa}{2}\right)^{2} \frac{\langle 3|1| 4]^{4}}{s^{2}}\left[\frac{i}{t-m^{2}}+\frac{i}{u-m^{2}}\right], \\
& \mathcal{A}_{\mathrm{EH}}^{(0)}\left(1^{\phi}, 2^{\phi}, 3^{++}, 4^{++}\right)=-\left(\frac{\kappa}{2}\right)^{2} m^{4} \frac{[34]^{2}}{\langle 34\rangle^{2}}\left[\frac{i}{t-m^{2}}+\frac{i}{u-m^{2}}\right],
\end{aligned}
$$

The computation of the four-point one-loop amplitude without helicity flip in the eikonal approximation (2.4) was performed in [13], with the result

$$
\begin{aligned}
\mathcal{A}_{\mathrm{EH}}^{(1)}\left(1^{\phi}, 2^{\phi}, 3^{--}, 4^{++}\right) \simeq & \mathcal{N}_{h}\left(\frac{\kappa}{2}\right)^{4}\left[(2 m \omega)^{4}\left(I_{4}(s, t ; m)+I_{4}(s, u ; m)\right)-15\left(m^{2} \omega\right)^{2} I_{3}(s ; m)\right. \\
& \left.+(4 m \omega)^{2} s I_{3}(s)-\frac{29}{2}(m \omega)^{2} I_{2}(s)\right],
\end{aligned}
$$

\footnotetext{
${ }^{3}$ We recall that $s=-|\vec{q}|^{2}$ where $\vec{q}$ is the momentum exchange between the classical source and the graviton.

${ }^{4}$ If the two particles are identical this introduces the correct Bose symmetry factor of $1 / 2$; if they are different this takes into account that the internal particles are not colour ordered, hence summing over two possible internal helicity assignments would lead to double counting, compensated by the factor of $1 / 2$.

${ }^{5}$ See for instance $[60,107]$.
} 


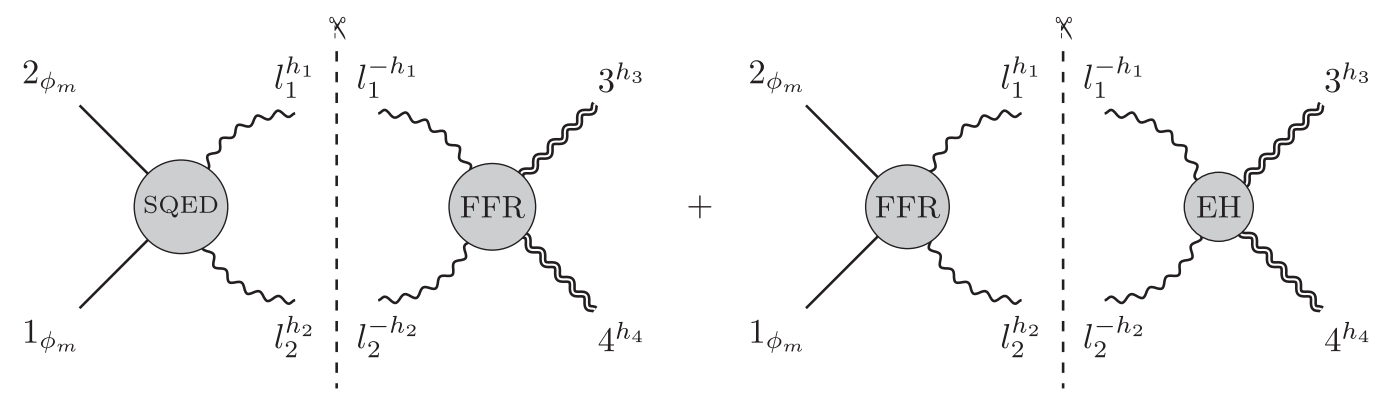

FIG. 2. The two-particle cut diagrams in the $s=-\vec{q}^{2}$-channel of the graviton deflection angle in the presence of an FFR interaction. The internal lines are photons. The first diagram is proportional to $\kappa^{2} e^{2}$ and is only nonvanishing for $h_{1}=h_{2}$ for the internal photons. The second diagram is proportional to $\kappa^{4}$, it is nonvanishing when $h_{4}=-h_{3}$ and $h_{2}=-h_{1}$ thus it contributes solely to the helicitypreserving configuration. Also, it only produces quantum corrections (bubble integrals) with coefficients that vanish in the case of fourdimensional external kinematics.

where

$$
\mathcal{N}_{h}:=\left(\frac{\langle 3|2| 4]}{2 m \omega}\right)^{4}
$$

is a pure phase, with $\mathcal{N}_{h} \rightarrow 1$ in the eikonal approximation. We have also computed the new amplitude with helicity flip in the same approximation, with the result

$$
\mathcal{A}_{\mathrm{EH}}^{(1)}\left(1^{\phi}, 2^{\phi}, 3^{++}, 4^{++}\right) \simeq\left(\frac{\kappa}{2}\right)^{4} \frac{[34]^{2}}{\langle 34\rangle^{2}}\left(m^{2} s\right)^{2}\left[I_{4}(s, t ; m)+I_{4}(s, u ; m)\right]
$$

\section{B. Four-point scalar/graviton scattering in $\mathbf{E H}+\boldsymbol{R}^{3}$}

We now consider the amplitudes with addition of the $R^{3}$ interaction in (1.1): the helicity-preserving amplitude at treelevel is vanishing

$$
\mathcal{A}_{R^{3}}^{(0)}\left(1^{\phi}, 2^{\phi}, 3^{--}, 4^{++}\right)=0
$$

while the helicity-flip amplitude is [60]

$$
\mathcal{A}_{R^{3}}^{(0)}\left(1^{\phi}, 2^{\phi}, 3^{++}, 4^{++}\right)=i\left(\frac{\kappa}{2}\right)^{2}\left(\frac{\alpha^{\prime}}{4}\right)^{2}[34]^{4} \frac{\left(t-m^{2}\right)\left(u-m^{2}\right)}{s} .
$$

At one loop, the result of [60] for the no-flip amplitude gives:

$$
\begin{aligned}
\mathcal{A}_{R^{3}}^{(1)}\left(1^{\phi}, 2^{\phi}, 3^{--}, 4^{++}\right) \simeq & \left(\frac{\kappa}{2}\right)^{4}\left(\frac{\alpha^{\prime}}{4}\right)^{2} \mathcal{N}_{h}\left[(m s)^{4}\left(I_{4}(s, t ; m)+I_{4}(s, u ; m)\right)+\left(m^{2} s \omega\right)^{2} I_{3}(s ; m)\right. \\
& \left.+\frac{3}{2}(m s \omega)^{2} I_{2}(s)\right]
\end{aligned}
$$

where $\mathcal{N}_{h}$ is defined in (3.3). The one-loop amplitude with helicity flip requires a new computation and the result in the eikonal approximation is

$$
\begin{aligned}
\mathcal{A}_{R^{3}}^{(1)}\left(1^{\phi}, 2^{\phi}, 3^{++}, 4^{++}\right) \simeq & \left(\frac{\kappa}{2}\right)^{4}\left(\frac{\alpha^{\prime}}{4}\right)^{2}[34]^{4}\left[(2 m \omega)^{4}\left(I_{4}(s, t ; m)+I_{4}(s, u ; m)\right)\right. \\
& \left.-13\left(m^{2} \omega\right)^{2} I_{3}(s ; m)+16(m \omega)^{2} s I_{3}(s)+\frac{153}{10}(m \omega)^{2} I_{2}(s)\right] .
\end{aligned}
$$




\section{Four-point scalar/graviton scattering in $\mathbf{E H}+\boldsymbol{R}^{4}$}

In this section we consider the addition of an $R^{4}$ interaction to the EH action. Such interaction affects the two-scalar two-graviton amplitude at one loop and thus contributes to graviton deflection and time delay at order $G^{2}$. In order to build this amplitude using the unitaritybased method we first need to find out the expression for the four-graviton tree-level amplitudes in the $R^{4}$ theory. We do so here starting from the Lagrangian in (1.3) in order to make contact with the notation of [58]; in Appendix D we present an alternative derivation only relying on little-group considerations and dimensional analysis, which does not require writing down any Lagrangian.

Deriving the four-graviton amplitudes from (1.3) is straightforward-we simply have to replace the four Riemann tensors in each term by their linearized form corresponding to the four on-shell gravitons. For particle $i$ the well-known expression in momentum space is

$$
R(i)_{\mu \nu \rho \sigma}=\frac{1}{2} F(i)_{\mu \nu} F(i)_{\rho \sigma}
$$

where

$$
F(i)_{\mu \nu}=p_{i \mu} \varepsilon_{i \nu}-p_{i \nu} \varepsilon_{i \mu} .
$$

Since we are interested in helicity amplitudes, we choose the field strengths $F(i)$ to be self-dual (negative helicity) or anti-self-dual (positive helicity), hence in spinor-helicity formalism their form is

$$
\begin{aligned}
F(i)_{\mathrm{SD} \alpha \dot{\alpha} \beta \dot{\beta}} & =-\sqrt{2} \lambda_{i \alpha} \lambda_{i \beta} \epsilon_{\dot{\alpha} \dot{\beta}} \quad \text { and } \\
F(i)_{\mathrm{ASD} \alpha \dot{\alpha} \beta \dot{\beta}} & =-\sqrt{2} \tilde{\lambda}_{i \dot{\alpha}} \tilde{\lambda}_{i \dot{\beta}} \epsilon_{\alpha \beta} .
\end{aligned}
$$

The building blocks in (1.4) are bilinear in Riemann tensors, and take the form

$$
\mathcal{C} \simeq\left(F(i)_{(\mathrm{A}) \mathrm{SD}} \cdot F(j)_{(\mathrm{A}) \mathrm{SD}}\right)^{2},
$$

and

$\tilde{\mathcal{C}} \simeq\left(F(i)_{(\mathrm{A}) \mathrm{SD}} \cdot F(j)_{(\mathrm{A}) \mathrm{SD}}\right)\left(F(i)_{(\mathrm{A}) \mathrm{SD}} \cdot \frac{1}{i} * F(j)_{(\mathrm{A}) \mathrm{SD}}\right)$,

where - denotes Lorentz contractions and $*$ denotes the usual Hodge dual which acts on the (anti-)selfdual field strengths as $* F_{\mathrm{SD}}=F_{\mathrm{SD}}$ and $* F_{\mathrm{ASD}}=-F_{\mathrm{ASD}}$. Furthermore, given the form (3.11) these expressions are only nonvanishing if both particles $i$ and $j$ have the same helicity. In summary, if both gravitons have negative helicity (SD field strength) we have

$$
\mathcal{C}=i \tilde{\mathcal{C}}=\frac{1}{2}\langle i j\rangle^{4},
$$

while if both gravitons have positive helicity (ASD field strength) we have

$$
\mathcal{C}=-i \tilde{\mathcal{C}}=\frac{1}{2}[i j]^{4} .
$$

With these results one easily arrives at

$$
\begin{aligned}
& \mathcal{A}_{R^{4}}^{(0)}\left(1^{++}, 2^{++}, 3^{++}, 4^{++}\right)=i \beta^{+}\left(\frac{\kappa}{2}\right)^{2}\left([12]^{4}[34]^{4}+[13]^{4}[24]^{4}+[14]^{4}[23]^{4}\right), \\
& \mathcal{A}_{R^{4}}^{(0)}\left(1^{--}, 2^{--}, 3^{--}, 4^{--}\right)=i \beta^{-}\left(\frac{\kappa}{2}\right)^{2}\left(\langle 12\rangle^{4}\langle 34\rangle^{4}+\langle 13\rangle^{4}\langle 24\rangle^{4}+\langle 14\rangle^{4}\langle 23\rangle^{4}\right), \\
& \mathcal{A}_{R^{4}}^{(0)}\left(1^{++}, 2^{++}, 3^{--}, 4^{--}\right)=i \tilde{\beta}\left(\frac{\kappa}{2}\right)^{2}[12]^{4}\langle 34\rangle^{4},
\end{aligned}
$$

with

$$
\begin{gathered}
\beta^{+}=4\left(\beta_{1}+\frac{i}{2} \beta_{2}-\beta_{3}\right), \\
\beta^{-}=4\left(\beta_{1}-\frac{i}{2} \beta_{2}-\beta_{3}\right), \\
\tilde{\beta}=4\left(\beta_{1}+\beta_{3}\right) .
\end{gathered}
$$

Note that if we do not allow the parity-odd coupling $\left(\beta_{2}=0\right)$, then the coefficient of the all-plus and all-minus amplitudes are the same $\beta^{+}=\beta^{-}:=\beta$. 
The next step is to carry out one-loop amplitude calculations in the eikonal approximation, as done in previous sections. The result for the relevant amplitudes is:

$$
\begin{aligned}
& \mathcal{A}_{R^{4}}^{(1)}\left(1^{\phi}, 2^{\phi}, 3^{--}, 4^{++}\right) \simeq-\mathcal{N}_{h} \tilde{\beta}\left(\frac{\kappa}{2}\right)^{4} s^{2}\left[\frac{35}{4}(m \omega)^{4} I_{3}(s ; m)+\frac{93}{8}\left(m \omega^{2}\right)^{2} I_{2}(s)\right], \\
& \mathcal{A}_{R^{4}}^{(1)}\left(1^{\phi}, 2^{\phi}, 3^{++}, 4^{++}\right) \simeq-\beta^{+}\left(\frac{\kappa}{2}\right)^{4}[34]^{4}\left[\frac{3}{4}(m \omega)^{4} I_{3}(s ; m)+\frac{55}{24}\left(m \omega^{2}\right)^{2} I_{2}(s)\right], \\
& \mathcal{A}_{R^{4}}^{(1)}\left(1^{\phi}, 2^{\phi}, 3^{--}, 4^{--}\right) \simeq-\beta^{-}\left(\frac{\kappa}{2}\right)^{4}\langle 34\rangle^{4}\left[\frac{3}{4}(m \omega)^{4} I_{3}(s ; m)+\frac{55}{24}\left(m \omega^{2}\right)^{2} I_{2}(s)\right],
\end{aligned}
$$

where $\mathcal{N}_{h}$ was introduced in (3.3).

\section{Scattering with the FFR interaction}

The last interaction we wish to consider is the FFR term in (1.1). From an on-shell point of view this is the simplest nonminimal modification of the coupling of photons to gravity. As we will show below this leads to new corrections to the bending and time delay/advance of light and graviton propagation in the background of a very massive scalar particle.

This new interaction modifies the three-point twophoton/one-graviton amplitude:

$$
\mathcal{A}_{\mathrm{FFR}}^{(0)}\left(1^{+}, 2^{+}, 3^{++}\right)=i\left(\frac{\kappa}{2}\right)\left(\frac{\alpha_{\gamma}}{4}\right)[13]^{2}[23]^{2},
$$

which we will now use to construct the relevant amplitudes at tree level and one loop to compute deflection angles and time delay in the presence of this interaction. Note that this amplitude is determined by its helicity structure and dimensional analysis up to a normalization which we fixed from the Feynman rule (B3) following from our action (1.1).

\section{Relevant amplitudes for graviton deflection}

Using factorization and Feynman diagrams we have computed the four-point amplitudes relevant for graviton deflection from a massive charged source (such as a charged black hole). The new FFR interaction involves two photons and one graviton, hence one cannot generate a tree-level correction to the amplitude with two scalars and two gravitons. The first corrections arise at one loop, from the cut diagrams in Fig. 2.

For the cut diagram on the left-hand side of the figure, we need the tree-level scalar QED amplitude with two photons and two massive scalars [9]

$\mathcal{A}_{\mathrm{SQED}}^{(0)}\left(1^{\phi}, 2^{\phi}, 3^{+}, 4^{+}\right)=Q^{2} m^{2} \frac{[34]^{2}}{s}\left(\frac{i}{t-m^{2}}+\frac{i}{u-m^{2}}\right)$,

along with the modification to the two-graviton/two-photon amplitudes arising from the FFR coupling for both helicity configurations of the graviton: no flip,
$\mathcal{A}_{\mathrm{FFR}}^{(0)}\left(1^{+}, 2^{+}, 3^{--}, 4^{++}\right)=-i\left(\frac{\kappa}{2}\right)^{2}\left(\frac{\alpha_{\gamma}}{4}\right)[12]^{2} \frac{\langle 3|1| 4]^{4}}{s t u}$,

or flipped,

$$
\begin{aligned}
& \mathcal{A}_{\mathrm{FFR}}^{(0)}\left(1^{+}, 2^{+}, 3^{++}, 4^{++}\right) \\
& \quad=i\left(\frac{\kappa}{2}\right)^{2}\left(\frac{\alpha_{\gamma}}{4}\right)\left(\frac{[13]^{2}[34]^{2}[42]^{2}}{s_{13}}+\frac{[23]^{2}[34]^{2}[41]^{2}}{s_{23}}\right) .
\end{aligned}
$$

Both amplitudes can be computed with on-shell techniques. Specifically, (3.23) can be constructed using BCFW recursion relations [108] by shifting appropriately the graviton momenta, while it is easy to verify [109] that (3.24) can be derived via an (holomorphic) all-line shift.

Note that the cut is nonvanishing only in the singlet configuration (internal photons with the same helicities). This is because the four-point amplitude with two photons and two gravitons induced by the FFR interaction is nonvanishing only for same-helicity photons.

We now move to the cut diagram on the right-hand side of Fig. 2. The two-photon/two-graviton EH amplitude only exists in the configuration where the gravitons and the photons have opposite helicity (see for instance [11]),

$\mathcal{A}_{\mathrm{EH}}^{(0)}\left(1^{+}, 2^{-}, 3^{++}, 4^{--}\right)=-i\left(\frac{\kappa}{2}\right)^{2}[13]^{2}\langle 24\rangle^{2} \frac{\langle 4|1| 3]^{2}}{s t u}$,

and thus it contributes only in the helicity-preserving process. Hence, in order to compute the cut we will only need the following two-scalar/two-photon amplitude involving an FFR interaction:

$$
\mathcal{A}_{\mathrm{FFR}}^{(0)}\left(1^{\phi}, 2^{\phi}, 3^{-}, 4^{+}\right)=-i\left(\frac{\kappa}{2}\right)^{2}\left(\frac{\alpha_{\gamma}}{4}\right)\langle 3|1| 4]^{2} .
$$

Performing the calculation, it turns out that the right-hand side of Fig. 2 does not produce any nonanalytic term with an $s$-channel discontinuity when external kinematics are considered to be strictly four-dimensional. 
Following the above considerations, the one-loop amplitudes in the eikonal limit can be computed entirely from the lhs of Fig. 2, and are found to be

$$
\begin{aligned}
\mathcal{A}_{\mathrm{FFR}}^{(1)}\left(1^{\phi}, 2^{\phi}, 3^{--}, 4^{++}\right) \simeq & -\mathcal{N}_{h} Q^{2}\left(\frac{\kappa}{2}\right)^{2}\left(\frac{\alpha_{\gamma}}{4}\right) s\left[(m s)^{2}\left(I_{4}(s, t ; m)+I_{4}(s, u ; m)\right)\right. \\
& \left.+(m \omega)^{2} I_{3}(s ; m)+\frac{3}{4} \frac{s^{3}}{\omega^{2}} I_{3}(s)+\frac{3}{2} \omega^{2} I_{2}(s)\right], \\
\mathcal{A}_{\mathrm{FFR}}^{(1)}\left(1^{\phi}, 2^{\phi}, 3^{++}, 4^{++}\right)= & Q^{2}\left(\frac{\kappa}{2}\right)^{2}\left(\frac{\alpha_{\gamma}}{4}\right) m^{2}[34]^{4} I_{3}(s ; m),
\end{aligned}
$$

where again $\mathcal{N}_{h}$ is the phase defined in (3.3), and $Q$ denotes the charge of the classical source (the black hole).

\section{Relevant amplitudes for photon deflection}

It is interesting to study how this new FFR interaction affects the bending and time delay/advance of light. In order to do so, we now review the known two-scalar/twophoton amplitudes for minimally coupled photons [11], and present the new corresponding amplitudes induced by the FFR interaction, both at tree and one-loop level.
In the following we consider processes where the internal legs are gravitons. In the EH theory, for the two-photon two-scalar process, only the helicity-preserving amplitude is nonvanishing, ${ }^{6}$ both at tree level

$$
\mathcal{A}_{\mathrm{EH}}^{(0)}\left(1^{\phi}, 2^{\phi}, 3^{-}, 4^{+}\right)=i\left(\frac{\kappa}{2}\right)^{2} \frac{\langle 3|1| 4]^{2}}{s},
$$

and at one loop [11],

$$
\begin{aligned}
\mathcal{A}_{\mathrm{EH}}^{(1)}\left(1^{\phi}, 2^{\phi}, 3^{-}, 4^{+}\right) \simeq & -\mathcal{N}_{\gamma}\left(\frac{\kappa}{2}\right)^{4}\left[(2 m \omega)^{4}\left(I_{4}(s, t ; m)+I_{4}(s, u ; m)\right)-15\left(m^{2} \omega\right)^{2} I_{3}(s ; m)\right. \\
& \left.+3 s(2 m \omega)^{2} I_{3}(s)-\frac{161}{30}(m \omega)^{2} I_{2}(s)\right],
\end{aligned}
$$

where the phase factor $\mathcal{N}_{\gamma}$ is

$$
\mathcal{N}_{\gamma}=\left(\frac{\langle 3|1| 4]}{2 m \omega}\right)^{2} \simeq-1
$$

We now discuss the corrections to the two-scalar two-photon amplitudes arising from one insertion of the FFR interaction. These come from a single graviton exchange between a minimally coupled scalar and the FFR three-point vertex. At tree level, only the helicity-flip amplitude

$$
\mathcal{A}_{\mathrm{FFR}}^{(0)}\left(1^{\phi}, 2^{\phi}, 3^{+}, 4^{+}\right)=-i\left(\frac{\kappa}{2}\right)^{2}\left(\frac{\alpha_{\gamma}}{4}\right)[34]^{2}\left[\frac{\left(t-m^{2}\right)\left(u-m^{2}\right)}{s}+m^{2}\right],
$$

contributes in the eikonal approximation, while the no-flip amplitude, already quoted in (3.26), is a contact term that is subleading in the eikonal limit (it does not have a pole in $s=-|\vec{q}|^{2}$ ).

Moving to one loop, the relevant two-particle cuts for the $(++)$ configuration are shown in Fig. 3 . We find that the amplitude with photons in the $(++)$ helicity configuration in the eikonal approximation is

$$
\begin{aligned}
\mathcal{A}_{\mathrm{FFR}}^{(1)}\left(1^{\phi}, 2^{\phi}, 3^{+}, 4^{+}\right) \simeq & -\left(\frac{\kappa}{2}\right)^{4}\left(\frac{\alpha_{\gamma}}{4}\right)[34]^{2}\left[(2 m \omega)^{4}\left(I_{4}(s, t ; m)+I_{4}(s, u ; m)\right)\right. \\
& \left.-15\left(m^{2} \omega\right)^{2} I_{3}(s ; m)+3 s(2 m \omega)^{2} I_{3}(s)+\frac{3}{10}(m \omega)^{2} I_{2}(s)\right],
\end{aligned}
$$

\footnotetext{
${ }^{6}$ Indeed, one can check that in four dimensions the Feynman rule for two same-helicity (on-shell) photons and one off-shell graviton $h$ is zero: $V^{\mu \nu}\left(1^{ \pm}, 2^{ \pm}, 3^{h}\right)=0$, where $V^{\mu \nu}$ is given in (B2).
} 


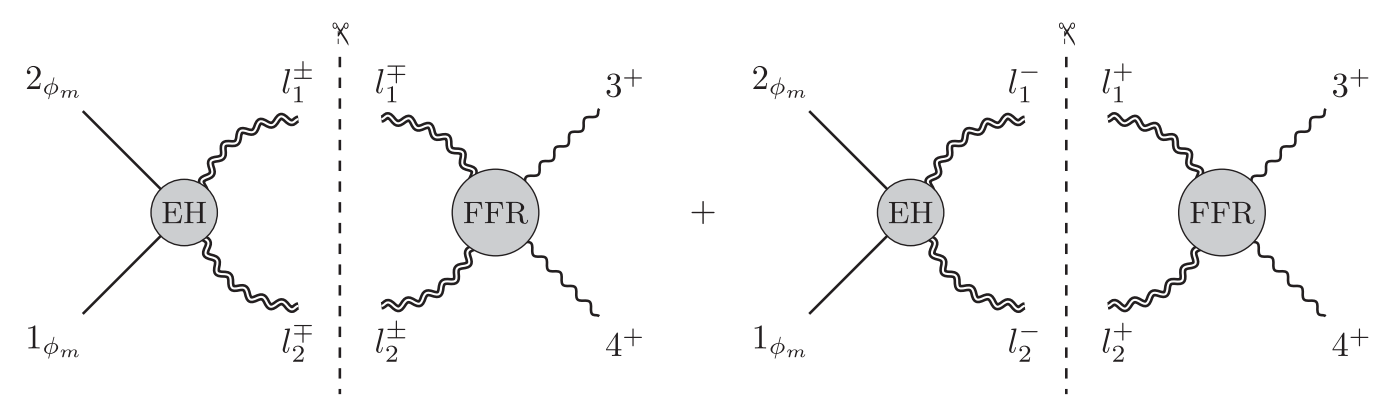

FIG. 3. The two-particle cut diagrams in the $s=-|\vec{q}|^{2}$-channel contributing to photon deflection to first order in the FFR interaction. We only show the helicity-flip configuration since the helicity-preserving cuts vanish. The cut diagram on the rhs of the figure only contributes terms which are subleading in the eikonal limit.

while the amplitude with photons in the $(+-)$ helicity configuration vanishes:

$$
\mathcal{A}_{\mathrm{FFR}}^{(1)}\left(1^{\phi}, 2^{\phi}, 3^{-}, 4^{+}\right)=0 .
$$

\section{EIKONAL PHASE MATRIX, DEFLECTION ANGLE AND TIME DELAY}

In the previous section we have derived the relevant tree and one-loop amplitudes which we will now use to extract the deflection angle and time delay up to 2PM order (or $\mathcal{O}\left(G^{2}\right)$ ) generated by the addition of the various couplings in (1.1). The key quantity is the eikonal phase matrix $\delta$, to be introduced below, of which we will compute the leading, $\delta_{0}$, and subleading contributions, $\delta_{1}$. As an important consistency check we will confirm that the leading-energy contribution of the one-loop amplitudes captures the required exponentiation of the leading-order eikonal phase matrix $\delta_{0}$.

In the following we focus on the classical contribution to $\delta$. We stress that for the cases we consider, $\delta$ will be a $2 \times 2$ matrix: the diagonal entries correspond to the two amplitudes $\mathcal{A}\left(1^{\phi}, 2^{\phi}, 3^{h_{1}}, 4^{h_{2}}\right)$ where the helicity of the massless particle is not flipped (which in our all-outgoing convention corresponds to $h_{1}=-h_{2}$ ), while the offdiagonal ones correspond to the two helicity-flip processes (with $h_{1}=h_{2}$ ).

As a final comment, we note that the combined effect of the interactions in (1.1) is simply the sum of the contributions of each interaction treated independently; hence we will study them separately, and begin our discussion by reviewing the computation in $\mathrm{EH}$ gravity.

\section{A. Graviton deflection angle and time delay in Einstein-Hilbert gravity}

\section{Leading eikonal}

The relevant tree-level amplitudes in EH gravity are given in (3.1). In the eikonal approximation (2.4) they become

$$
\begin{aligned}
& \mathcal{A}_{\mathrm{EH}}^{(0)}\left(1^{\phi}, 2^{\phi}, 3^{--}, 4^{++}\right) \simeq i\left(\frac{\kappa}{2}\right)^{2} \frac{(2 m \omega)^{2}}{\vec{q}^{2}}, \\
& \mathcal{A}_{\mathrm{EH}}^{(0)}\left(1^{\phi}, 2^{\phi}, 3^{++}, 4^{++}\right) \simeq i\left(\frac{\kappa}{2}\right)^{2} \frac{m^{2}}{(2 \omega)^{2}} \frac{q^{4}}{\vec{q}^{2}} \simeq 0,
\end{aligned}
$$

where the second amplitude is subleading compared to the first.

The amplitudes in impact parameter space are obtained from those in momentum space using (2.9). To compute them, we will use repeatedly the result

$f(p, d):=\int \frac{d^{d} q}{(2 \pi)^{d}} e^{i \vec{q} \cdot \vec{b}}|\vec{q}|^{p}=\frac{2^{p} \pi^{-d / 2} \Gamma\left(\frac{d+p}{2}\right)}{\Gamma\left(-\frac{p}{2}\right)} \frac{1}{b^{d+p}}$,

where $b:=|\vec{b}|$. We then have

$\left.\tilde{\mathcal{A}}_{\mathrm{EH}}^{(0)}\left(1^{\phi}, 2^{\phi}, 3^{--}, 4^{++}\right)\right|_{\omega}=i\left(\frac{\kappa}{2}\right)^{2} \frac{m \omega}{4 \pi^{\frac{D-2}{2}}} \Gamma\left(\frac{D}{2}-2\right) \frac{1}{b^{D-4}}$,

$\left.\tilde{\mathcal{A}}_{\mathrm{EH}}^{(0)}\left(1^{\phi}, 2^{\phi}, 3^{++}, 4^{++}\right)\right|_{\omega}=0$,

therefore the leading eikonal phase matrix is

$$
\begin{aligned}
\delta_{0, \mathrm{EH}} & =\left(\frac{\kappa}{2}\right)^{2}(m \omega) f(-2, D-2) \mathbb{1}_{2} \\
& \simeq-\left(\frac{\kappa}{2}\right)^{2} \frac{m \omega}{2 \pi}\left[\frac{1}{4-D}+\log b\right] \mathbb{1}_{2}+\cdots,
\end{aligned}
$$

where we omitted terms of $\mathcal{O}(D-4)$ and finite terms which do not depend on $\vec{b}$.

Next we consider the one-loop amplitudes (3.2) and (3.4). In order to check exponentiation (2.15) we only keep terms that are leading in energy in the eikonal approximation, i.e., $\mathcal{O}\left(\omega^{3}\right)$ in momentum space (or $\mathcal{O}\left(\omega^{2}\right)$ in impact parameter space). These are 


$$
\begin{aligned}
\left.\mathcal{A}_{\mathrm{EH}}^{(1)}\left(1^{\phi}, 2^{\phi}, 3^{--}, 4^{++}\right)\right|_{\omega^{3}}= & \left(\frac{\kappa}{2}\right)^{4}(2 m \omega)^{4}\left[I_{4}(s, t ; m)\right. \\
& \left.+I_{4}(s, u ; m)\right], \\
\left.\mathcal{A}_{\mathrm{EH}}^{(1)}\left(1^{\phi}, 2^{\phi}, 3^{++}, 4^{++}\right)\right|_{\omega^{3}}= & 0,
\end{aligned}
$$

where the sum of the box integrals $I_{4}(s, t ; m)+I_{4}(s, u ; m)$ was evaluated in $D$ dimensions in [55] and is given in (A4). Transforming to impact parameter space, we have

$$
\begin{aligned}
& \left.\tilde{\mathcal{A}}_{\mathrm{EH}}^{(1)}\left(1^{\phi}, 2^{\phi}, 3^{--}, 4^{++}\right)\right|_{\omega^{2}} \\
& \quad=-\left(\frac{\kappa}{2}\right)^{4}(m \omega)^{2} \frac{2^{D-7} \Gamma(D-4)}{\pi^{\frac{D}{2}}(D-4) \Gamma(3-D / 2)} \frac{1}{b^{2 D-8}} .
\end{aligned}
$$

As expected from (2.15), we find that

$$
\begin{aligned}
& \left.\tilde{\mathcal{A}}_{\mathrm{EH}}^{(1)}\left(1^{\phi}, 2^{\phi}, 3^{--}, 4^{++}\right)\right|_{\omega^{2}} \\
& \quad=\frac{1}{2}\left[\left.\tilde{\mathcal{A}}_{\mathrm{EH}}^{(0)}\left(1^{\phi}, 2^{\phi}, 3^{--}, 4^{++}\right)\right|_{\omega}\right]^{2}+\mathcal{O}(D-4) .
\end{aligned}
$$

\section{Subleading eikonal}

In momentum space, the subleading contribution to the eikonal phase matrix is extracted from the $\mathcal{O}\left(\omega^{2}\right)$ contribution to the amplitude in (3.2)

$\left.\mathcal{A}_{\mathrm{EH}}^{(1)}\left(1^{\phi}, 2^{\phi}, 3^{--}, 4^{++}\right)\right|_{\omega^{2}}=\left(\frac{\kappa}{2}\right)^{4}\left(-15 m^{4} \omega^{2}\right) I_{3}(s ; m)$,

where $I_{3}(s ; m)$ is given in (A3), and as usual $s=-|\vec{q}|^{2}$. In the following we focus on the first term on the right-hand side of (A3), since the log term only contributes quantum corrections. Using

$$
\int \frac{d^{D-2} q}{(2 \pi)^{D-2}} e^{i \vec{q} \cdot \vec{b}}|\vec{q}|^{-1}=\frac{1}{2 \pi} \frac{1}{b}+\mathcal{O}(D-4),
$$

we obtain the subleading part of the amplitude in impact parameter space:

$$
\left.\tilde{\mathcal{A}}_{\mathrm{EH}}^{(1)}\left(1^{\phi}, 2^{\phi}, 3^{--}, 4^{++}\right)\right|_{\omega}=i\left(\frac{\kappa}{2}\right)^{4} \frac{15}{256 \pi} \frac{m^{2} \omega}{b},
$$

and finally, using (2.13), $\delta_{1}$ :

$$
\delta_{1, \mathrm{EH}}=\left(\frac{\kappa}{2}\right)^{4} \frac{15}{256 \pi} \frac{m^{2} \omega}{b} \mathbb{1}_{2} .
$$

The eikonal phase matrix up to one loop in EH is then given by

\footnotetext{
${ }^{7}$ Note that such a contribution is absent in (3.4).
}

$$
\begin{aligned}
\delta_{\mathrm{EH}}= & \delta_{0, \mathrm{EH}}+\delta_{1, \mathrm{EH}}+\cdots \\
= & -\left(\frac{\kappa}{2}\right)^{2} \frac{m \omega}{2 \pi}\left[\frac{1}{4-D}+\log b-\left(\frac{\kappa}{2}\right)^{2} \frac{15}{256 \pi} \frac{m}{b}\right] \mathbb{1}_{2} \\
& +\cdots
\end{aligned}
$$

Note that this matrix is proportional to the identity, since the polarization of the gravitons scattered by the classical source is unchanged. The deflection angle can now be extracted using (2.16). While the eigenvalues of $\delta$ are divergent in $D=4$, the corresponding deflection angle is finite:

$$
\begin{aligned}
\theta_{\mathrm{EH}} & =-\frac{1}{2 \pi}\left(\frac{\kappa}{2}\right)^{2} \frac{m}{b}\left[1+\left(\frac{\kappa}{2}\right)^{2} \frac{15}{128} \frac{m}{b}\right] \\
& =-\frac{4 G m}{b}\left(1+G \frac{15 \pi}{16} \frac{m}{b}\right) .
\end{aligned}
$$

This result agrees with the derivation of [13], and as expected matches the photon deflection angle [10,11], first computed by Einstein. ${ }^{8}$

Another quantity of interest which can be extracted from the eigenvalues of the eikonal matrix is the time delay. Using (2.17) applied to the leading eikonal phase (4.4), we get

$$
t_{\mathrm{EH}}=-\left(\frac{\kappa}{2}\right)^{2} \frac{m}{2 \pi}\left(\frac{1}{4-D}+\log b\right) .
$$

As is well known, in order to define the time delay in four dimensions we need to take the difference of two time delays as measured by an observer at $b$ and one at a much larger distance $b_{0} \gg b$ [87]. Doing so the pole in (4.14) drops out, and neglecting power-suppressed terms in $b_{0}$ one gets

$$
t_{\mathrm{EH}}=\left(\frac{\kappa}{2}\right)^{2} \frac{m}{2 \pi} \log \frac{b_{0}}{b}=4 G m \log \frac{b_{0}}{b},
$$

in agreement with [111]. Including now also the contribution from $\delta_{1}$, we arrive at the result

$$
\begin{aligned}
t_{\mathrm{EH}} & =\left(\frac{\kappa}{2}\right)^{2} \frac{m}{2 \pi}\left[\log \frac{b_{0}}{b}+\left(\frac{\kappa}{2}\right)^{2} \frac{15}{128} \frac{m}{b}\right] \\
& =4 G m\left[\log \frac{b_{0}}{b}+G \frac{15 \pi}{16} \frac{m}{b}\right] .
\end{aligned}
$$

In the next sections we compute the corrections $\Delta \theta_{X}$ and $\Delta t_{X}$ to the deflection angle (4.13) and time delay (4.15) in $\mathrm{EH}$ due to the inclusion of an interactions $X$ in (1.1). The complete deflection angle and time delay will then be $\theta_{\mathrm{EH}}+\Delta \theta_{X}$ and $t_{\mathrm{EH}}+\Delta t_{X}$.

\footnotetext{
${ }^{8}$ Initially up to a factor of two [110].
} 


\section{B. Graviton deflection angle and time delay in $\mathbf{E H}+\boldsymbol{R}^{3}$}

\section{Leading eikonal}

The relevant new amplitudes are obtained by evaluating (3.5) and (3.6) in the eikonal limit (2.4), with the result

$\mathcal{A}_{R^{3}}^{(0)}\left(1^{\phi}, 2^{\phi}, 3^{--}, 4^{++}\right)=0$,

$\mathcal{A}_{R^{3}}^{(0)}\left(1^{\phi}, 2^{\phi}, 3^{++}, 4^{++}\right) \simeq i\left(\frac{\kappa}{2}\right)^{2}\left(\frac{\alpha^{\prime}}{4}\right)^{2}(2 m \omega)^{2} \frac{q^{4}}{\vec{q}^{2}}$,

where from (2.7) we have $[34]^{4}=q^{4}$. In order to transform to impact parameter space we rewrite

$$
\vec{b} \cdot \vec{q}=\mathfrak{b} \bar{q}+\overline{\mathfrak{b}} q
$$

with $\mathfrak{b}:=\left(b_{1}+i b_{2}\right) / 2$, and $\overline{\mathfrak{b}}:=\left(b_{1}-i b_{2}\right) / 2$ (and we recall our previous definitions $\left.q=q_{1}+i q_{2}, \bar{q}=q_{1}-i q_{2}\right)$, from which $\mathfrak{b} \overline{\mathfrak{b}}=b^{2} / 4$. Then in $\vec{b}$-space we have

$$
\begin{aligned}
& \left.\tilde{\mathcal{A}}_{R^{3}}^{(0)}\left(1^{\phi}, 2^{\phi}, 3^{++}, 4^{++}\right)\right|_{\omega} \\
& =i\left(\frac{\kappa}{2}\right)^{2}\left(\frac{\alpha^{\prime}}{4}\right)^{2}(m \omega)\left(\frac{\partial}{\partial \overline{\mathfrak{b}}}\right)^{4} f(-2, D-2) \\
& =i\left(\frac{\kappa}{2}\right)^{2}\left(\frac{\alpha^{\prime}}{4}\right)^{2} \frac{(m \omega)}{\overline{\mathfrak{b}}^{4}} \xi f(-2, D-2),
\end{aligned}
$$

where

$$
\xi:=\left(\frac{D}{2}-2\right)\left(\frac{D}{2}-1\right)\left(\frac{D}{2}\right)\left(\frac{D}{2}+1\right) .
$$

Hence the leading eikonal phase matrix $\delta_{0}$, including the first contribution from the $R^{3}$ interaction, has the form

$$
\delta_{0}=\delta_{0, \mathrm{EH}}+\delta_{0, R^{3}},
$$

where $\delta_{0, \mathrm{EH}}$ is given in (4.4), and

$\delta_{0, R^{3}}=\left(\frac{\kappa}{2}\right)^{2}\left(\frac{\alpha^{\prime}}{4}\right)^{2}(m \omega)[\xi f(-2, D-2)]\left(\begin{array}{cc}0 & \overline{\mathfrak{b}}^{-4} \\ \mathfrak{b}^{-4} & 0\end{array}\right)$,

where we have used (2.12).

Moving on to one loop, from (3.7) and (3.8) we obtain

$$
\begin{aligned}
\left.\mathcal{A}_{R^{3}}^{(1)}\left(1^{\phi}, 2^{\phi}, 3^{--}, 4^{++}\right)\right|_{\omega^{3}}= & 0 \\
\left.\mathcal{A}_{R^{3}}^{(1)}\left(1^{\phi}, 2^{\phi}, 3^{++}, 4^{++}\right)\right|_{\omega^{3}}= & \left(\frac{\kappa}{2}\right)^{4}\left(\frac{\alpha^{\prime}}{4}\right)^{2}[34]^{4}(2 m \omega)^{4} \\
& \times\left[I_{4}(s, t)+I_{4}(s, u)\right] .
\end{aligned}
$$

Transforming to impact parameter space, and using (A4), we arrive at

$$
\begin{aligned}
\left.\tilde{\mathcal{A}}_{R^{3}}^{(1)}\left(1^{\phi}, 2^{\phi}, 3^{++}, 4^{++}\right)\right|_{\omega^{2}} & =-\left(\frac{\kappa}{2}\right)^{4}\left(\frac{\alpha^{\prime}}{4}\right)^{2} \frac{(m \omega)^{2}}{2 \pi} \frac{1}{D-4}\left(\frac{\partial}{\partial \overline{\mathfrak{b}}}\right)^{4} f(D-6, D-2) \\
& =-\left(\frac{\kappa}{2}\right)^{4}\left(\frac{\alpha^{\prime}}{4}\right)^{2} \frac{(m \omega)^{2}}{2 \pi \overline{\mathfrak{b}}^{4}} \frac{\xi^{\prime}}{D-4} f(D-6, D-2),
\end{aligned}
$$

where

$$
\xi^{\prime}:=(D-4)(D-3)(D-2)(D-1) .
$$

The leading one-loop amplitude matrix in the eikonal approximation is then found to be

$$
\mathcal{A}_{\omega^{2}}^{(1)}=-\left(\frac{\kappa}{2}\right)^{4}(m \omega)^{2} \frac{f(D-6, D-2)}{2 \pi(D-4)}\left(\begin{array}{cc}
1 & \left(\frac{\alpha^{\prime}}{4}\right)^{2} \frac{\xi^{\prime}}{\mathfrak{b}^{4}} \\
\left(\frac{\alpha^{\prime}}{4}\right)^{2} \frac{\xi^{\prime}}{\mathfrak{b}^{4}} & 1
\end{array}\right) .
$$

One can then check the matrix relation

$$
\mathcal{A}_{\omega^{2}}^{(1)}=-\frac{1}{2}\left(\delta_{0}\right)^{2}+\mathcal{O}(D-4),
$$

in agreement with (2.15). In writing (4.27) we have used that,

$$
\left(\delta_{0}\right)^{2}=\left(\frac{\kappa}{2}\right)^{4}(m \omega)^{2}[f(-2, D-2)]^{2}\left(\begin{array}{cc}
1 & \left(\frac{\alpha^{\prime}}{4}\right)^{2} \frac{2 \xi}{\overline{\mathfrak{b}}^{4}} \\
\left(\frac{\alpha^{\prime}}{4}\right)^{2} \frac{2 \xi}{\mathfrak{b}^{4}} & 1
\end{array}\right),
$$

up to and including $\mathcal{O}\left(\left(\alpha^{\prime} / 4\right)^{2}\right)$.

Finally we compute the eigenvalues of the matrix $\delta_{0}$ in (4.21). Using

$$
\xi f(-2, D-2)=\frac{3}{2 \pi}+\mathcal{O}(D-4),
$$

we can rewrite it as

$$
\delta_{0}=\left(\frac{\kappa}{2}\right)^{2} \frac{m \omega}{2 \pi}\left(\begin{array}{cc}
-\frac{1}{2 \epsilon}-\log b & \left(\frac{\alpha^{\prime}}{4}\right)^{2} \frac{3}{\mathfrak{b}^{4}} \\
\left(\frac{\alpha^{\prime}}{4}\right)^{2} \frac{3}{\mathfrak{b}^{4}} & -\frac{1}{2 \epsilon}-\log b
\end{array}\right),
$$

whose eigenvalues are 


$$
\delta_{0}^{(1,2)}=\left(\frac{\kappa}{2}\right)^{2} \frac{m \omega}{2 \pi}\left[-\frac{1}{2 \epsilon}-\log b \pm\left(\frac{\alpha^{\prime}}{4}\right)^{2} \frac{48}{b^{4}}\right] .
$$

Following identical steps to those leading from (4.12) to (4.16), one obtains for the time delay at $\mathcal{O}(G)$

$$
t_{\mathrm{EH}+R^{3}}=4 G m\left[\log \frac{b_{0}}{b} \pm\left(\frac{\alpha^{\prime}}{4}\right)^{2} \frac{48}{b^{4}}\right],
$$

where $G=\kappa^{2} /(32 \pi)$. For sufficiently small $b$ the eigenvalue with the choice of negative sign may become negative, leading to a time advance. We will come back to the time delay computation and add $\mathcal{O}\left(G^{2}\right)$ corrections in Section IV B 4.

\section{Comparison to the work of [87]}

The time advance due to $R^{3}$ terms was first discovered in [87], from which it was argued that the only way to avoid causality violations is to embed the $R^{3}$ theory into an appropriate ultraviolet completion-in other words a consistent ultraviolet completion of gravitational theories with an $R^{3}$ interaction requires the addition of an infinite tower of massive particles with higher spins. Here we wish to briefly compare our results to theirs.

The authors of [87] considered the interaction of a graviton with the background produced by a coherent state of massless particles, and computed the eikonal phase in order to obtain the Shapiro time delay. The coherent state simulates a large number of successive interactions of the graviton with a single weakly coupled particle, each instance being considered as independent and contributing with a small amount to the total phase shift. It is then observed that the presence of the $R^{3}$ coupling, which modifies the three-point graviton amplitude, leads to nondegenerate eigenvalues of the eikonal phase matrix.

Concretely, it is interesting to compare the eigenvalues (4.31) of the leading eikonal phase matrix (4.21). Pleasingly, these eigenvalues turn out to be identical ${ }^{9}$ to the eigenvalues (3.22) of [87], upon replacing $m \omega \rightarrow \omega^{2}$. This is due to the fact that we consider a different setup, with massless gravitons moving in the background produced by massive scalar objects of mass $m$. In both cases the time advance is induced by the novel three-graviton coupling generated by the $R^{3}$ interaction.

\section{Subleading eikonal}

We now go back to the one-loop amplitudes (3.7) and (3.8) and extract the triangle contributions which are the relevant terms contributing to the subleading eikonal matrix:

\footnotetext{
${ }^{9}$ Note that in (3.22) of [87] the $1 / \epsilon$ pole was not written explicitly. This pole does not affect either the time delay (4.32) or the particle bending angle. Our $1 / \epsilon$ pole corresponds to the $\log L$ term in [87], where $L$ is an infrared cutoff.
}

$$
\begin{aligned}
\left.\mathcal{A}_{R^{3}}^{(1)}\left(1^{\phi}, 2^{\phi}, 3^{--}, 4^{++}\right)\right|_{\omega^{2}}= & \left(\frac{\kappa}{2}\right)^{4}\left(\frac{\alpha^{\prime}}{4}\right)^{2} \\
& \times|\vec{q}|^{4} m^{4} \omega^{2} I_{3}(s ; m), \\
\left.\mathcal{A}_{R^{3}}^{(1)}\left(1^{\phi}, 2^{\phi}, 3^{++}, 4^{++}\right)\right|_{\omega^{2}}= & -13\left(\frac{\kappa}{2}\right)^{4}\left(\frac{\alpha^{\prime}}{4}\right)^{2} \\
& \times q^{4} m^{4} \omega^{2} I_{3}(s ; m) .
\end{aligned}
$$

We can now transform to impact parameter space, using

$$
\int \frac{d^{D-2} q}{(2 \pi)^{D-2}} e^{i \vec{q} \cdot \vec{b}}|\vec{q}|^{3}=\frac{9}{2 \pi} \frac{1}{b^{5}}+\mathcal{O}(D-4)
$$

$$
\left(\frac{\partial}{\partial \overline{\mathfrak{b}}}\right)^{4} \int \frac{d^{D-2} q}{(2 \pi)^{D-2}} e^{i \vec{q} \cdot \vec{b}}|\vec{q}|^{-1}=\frac{105}{32 \pi} \frac{1}{b} \frac{1}{\overline{\mathfrak{b}}^{4}}+\mathcal{O}(D-4)
$$

The amplitudes in impact parameter space then become

$$
\begin{aligned}
& \left.\tilde{\mathcal{A}}_{R^{3}}^{(1)}\left(1^{\phi}, 2^{\phi}, 3^{--}, 4^{++}\right)\right|_{\omega}=-i\left(\frac{\kappa}{2}\right)^{4}\left(\frac{\alpha^{\prime}}{4}\right)^{2} \frac{9}{256 \pi} \frac{m^{2} \omega}{b^{5}}, \\
& \left.\tilde{\mathcal{A}}_{R^{3}}^{(1)}\left(1^{\phi}, 2^{\phi}, 3^{++}, 4^{++}\right)\right|_{\omega}=i\left(\frac{\kappa}{2}\right)^{4}\left(\frac{\alpha^{\prime}}{4}\right)^{2} \frac{1365}{4096 \pi} \frac{m^{2} \omega}{b} \frac{1}{\overline{\mathfrak{b}}^{4}} .
\end{aligned}
$$

Using (2.12), we can extract the contribution of the $R^{3}$ interaction to the subleading eikonal matrix $\delta_{1}$ :

$$
\delta_{1, R^{3}}=\left(\frac{\kappa}{2}\right)^{4}\left(\frac{\alpha^{\prime}}{4}\right)^{2} \frac{1}{256 \pi} \frac{m^{2} \omega}{b}\left(\begin{array}{cc}
-\frac{9}{b^{4}} & \frac{1365}{16} \frac{1}{\mathfrak{b}^{4}} \\
\frac{1365}{16} \frac{1}{\mathfrak{b}^{4}} & -\frac{9}{b^{4}}
\end{array}\right) .
$$

\section{Deflection angle and time delay}

We can proceed similarly to the EH case. In the previous sections we showed that the $R^{3}$ interaction introduced offdiagonal terms, i.e., the helicity of the scattered graviton can change.

The eigenvalues of the leading and subleading eikonal matrices (4.22) and (4.37) are

$$
\begin{gathered}
\delta_{0, R^{3}}^{(1,2)}= \pm\left(\frac{\kappa}{2}\right)^{2}\left(\frac{\alpha^{\prime}}{4}\right)^{2} \frac{24}{\pi} \frac{m \omega}{b^{4}} \\
\delta_{1, R^{3}}^{(1,2)}=\left(\frac{\kappa}{2}\right)^{4}\left(\frac{\alpha^{\prime}}{4}\right)^{2} \frac{1}{256 \pi} \frac{m^{2} \omega}{b^{5}}(-9 \pm 1365) .
\end{gathered}
$$

Next we present the correction to the graviton deflection angle, both in terms of $\kappa$ and $G$ : 


$$
\begin{aligned}
\Delta \theta_{R^{3}}^{(1,2)}= & -\frac{1}{2 \pi}\left(\frac{\kappa}{2}\right)^{2}\left(\frac{\alpha^{\prime}}{4}\right)^{2} \frac{m}{b} \\
& \times\left[ \pm \frac{192}{b^{4}}+\frac{5}{128}(-9 \pm 1365)\left(\frac{\kappa}{2}\right)^{2} \frac{m}{b^{5}}\right] \\
= & -\frac{4 G m}{b}\left(\frac{\alpha^{\prime}}{4}\right)^{2}\left[ \pm \frac{192}{b^{4}}+\frac{5 \pi}{16}(-9 \pm 1365) \frac{G m}{b^{5}}\right] .
\end{aligned}
$$

The deflection involving a graviton whose helicity is preserved in the scattering process has already been studied in [60], instead the flipped helicity case is presented here for the first time.

Finally, for the time delay, proceeding as in Sec. IV A 2, and applying (2.17) to (4.38) and (4.39), we arrive at

$$
\begin{aligned}
\Delta t_{R^{3}}^{(1,2)}= & \left(\frac{\kappa}{2}\right)^{2}\left(\frac{\alpha^{\prime}}{4}\right)^{2} \frac{m}{2 \pi} \\
& \times\left[ \pm 48 \frac{1}{b^{4}}+\left(\frac{\kappa}{2}\right)^{2} \frac{1}{128} \frac{m}{b^{5}}(-9 \pm 1365)\right] \\
= & 4 G m\left(\frac{\alpha^{\prime}}{4}\right)^{2}\left[ \pm 48 \frac{1}{b^{4}}+\frac{\pi}{16}(-9 \pm 1365) \frac{G m}{b^{5}}\right]
\end{aligned}
$$

\section{Graviton deflection angle and time delay in $\mathbf{E H}+\boldsymbol{R}^{\mathbf{4}}$}

In this section we consider the deflection of gravitons induced by eight-derivative couplings in the Lagrangian, which we collectively denote as $R^{4}$. We will only consider the parity-even interactions in (1.3) in order to present more compact formulas, therefore we set $\beta_{2}=0$, and hence $\beta^{+}=\beta^{-}=\beta$ in (3.16) and (3.20). Furthermore, since these interactions do not produce a three-graviton vertex, it is impossible to build any tree-level two-scalar two-graviton amplitude involving $R^{4}$. Thus there is no tree-level (1PM) bending associated to the new term in the Lagrangian, and one has

$$
\delta_{0, R^{4}}=0
$$

and the leading contribution arises at 2PM order. Furthermore, since the $R^{4}$ term only produces a contact term four-graviton interaction, the resulting one-loop amplitudes does not contain any box integral. This is consistent with the absence of a tree-level contribution in (4.42) which, in the eikonal approximation, is expected to exponentiate, and would result at one loop in the appearance of box integrals. The same situation occurs for the graviton deflection due to FFR couplings discussed in Sec. IV D.

The relevant one-loop amplitudes are given in (3.20), and from the massive triangle contributions we extract the following results in the eikonal approximation:
$\left.\mathcal{A}_{R^{4}}^{(1)}\left(1^{\phi}, 2^{\phi}, 3^{--}, 4^{++}\right)\right|_{\omega^{4}}=i \tilde{\beta}\left(\frac{\kappa}{2}\right)^{4} \frac{35}{128} m^{3} \omega^{4}|\vec{q}|^{3}$,

$\left.\mathcal{A}_{R^{4}}^{(1)}\left(1^{\phi}, 2^{\phi}, 3^{++}, 4^{++}\right)\right|_{\omega^{4}}=i \beta\left(\frac{\kappa}{2}\right)^{4} \frac{3}{128} m^{3} \omega^{4} \frac{q^{4}}{|\vec{q}|}$,

which then translate in impact parameter space into

$$
\begin{aligned}
& \left.\tilde{\mathcal{A}}_{R^{4}}^{(1)}\left(1^{\phi}, 2^{\phi}, 3^{--}, 4^{++}\right)\right|_{\omega^{3}}=i \tilde{\beta}\left(\frac{\kappa}{2}\right)^{4} \frac{315}{512} \frac{m^{2} \omega^{3}}{2 \pi b^{5}} \\
& \left.\tilde{\mathcal{A}}_{R^{4}}^{(1)}\left(1^{\phi}, 2^{\phi}, 3^{++}, 4^{++}\right)\right|_{\omega^{3}}=i \beta\left(\frac{\kappa}{2}\right)^{4} \frac{315}{512} \frac{m^{2} \omega^{3}}{32 \pi b} \frac{1}{\overline{\mathfrak{b}}^{4}} .
\end{aligned}
$$

The subleading eikonal phase matrix resulting from the previous amplitudes is given by

$$
\delta_{1, R^{4}}=\left(\frac{\kappa}{2}\right)^{4} \frac{315}{512} \frac{m^{2} \omega^{3}}{2 \pi} \frac{1}{b}\left(\begin{array}{cc}
\tilde{\beta} \frac{1}{b^{4}} & \frac{\beta}{16} \frac{1}{\overline{\mathfrak{b}}^{4}} \\
\frac{\beta}{16} \frac{1}{\mathfrak{b}^{4}} & \tilde{\beta} \frac{1}{b^{4}}
\end{array}\right),
$$

whose eigenvalues are easily computed to be

$$
\delta_{1, R^{4}}^{(1,2)}=(\tilde{\beta} \pm \beta)\left(\frac{\kappa}{2}\right)^{4} \frac{315}{512} \frac{m^{2} \omega^{3}}{2 \pi} \frac{1}{b^{5}}
$$

Using (2.16) we can then extract the deflection angle

$$
\begin{aligned}
\Delta \theta_{R^{4}}^{(1,2)} & =-(\tilde{\beta} \pm \beta)\left(\frac{\kappa}{2}\right)^{4} \frac{1575}{512} \frac{m^{2} \omega^{2}}{2 \pi} \frac{1}{b^{6}} \\
& =-(\tilde{\beta} \pm \beta)(G m)^{2} \frac{1575 \pi}{16} \frac{\omega^{2}}{b^{6}}
\end{aligned}
$$

Similarly to the $\mathrm{EH}$ and the $R^{3}$ interaction we can extract the time delay arising from the $R^{4}$ interaction in (1.1), which in this case arises entirely from the subleading eikonal phase. Applying (2.17) to (4.46) we find

$$
\begin{aligned}
\Delta t_{R^{4}}^{(1,2)} & =(\tilde{\beta} \pm \beta)\left(\frac{\kappa}{2}\right)^{4} \frac{945}{512} \frac{m^{2} \omega^{2}}{2 \pi} \frac{1}{b^{5}} \\
& =(\tilde{\beta} \pm \beta)(G m)^{2} \frac{945 \pi}{16} \frac{\omega^{2}}{b^{5}} .
\end{aligned}
$$

We can express (4.47) and (4.48) in terms of the couplings introduced in (1.3), using (3.17), (3.18) and (3.19). In the parity-even theory $\left(\beta_{2}=0\right)$ we get $\beta+\tilde{\beta}=8 \beta_{1}$, and $\tilde{\beta}-\beta=8 \beta_{3}$. In order to avoid a potential time-advance and associated causality violation, we need to require

$$
\beta_{1}>0 \text { and } \beta_{3}>0 \text {. }
$$

Interestingly this positivity constraint is the same as derived from causality considerations in [96] and general $S$-matrix analyticity properties in [97]. 


\section{Graviton deflection angle and time delay in EH + FFR}

Next we focus our attention on graviton deflection in $\mathrm{EH}$ theory with the addition of an FFR coupling. As discussed in Sec. III D 1, at tree level there is no new two-scalar twograviton amplitude generated by this interaction, hence

$$
\delta_{0, \mathrm{FFR}}=0 .
$$

In order to compute the subleading eikonal phase matrix, we look at the massive triangle contribution to the one-loop amplitudes in (3.27),

$\left.\mathcal{A}_{\mathrm{FFR}}^{(1)}\left(1^{\phi}, 2^{\phi}, 3^{--}, 4^{++}\right)\right|_{\omega^{2}}=-i Q^{2}\left(\frac{\kappa}{2}\right)^{2}\left(\frac{\alpha_{\gamma}}{4}\right) \frac{m \omega^{2}}{32}|\vec{q}|$,

$\left.\mathcal{A}_{\mathrm{FFR}}^{(1)}\left(1^{\phi}, 2^{\phi}, 3^{++}, 4^{++}\right)\right|_{\omega^{2}}=0$.

Using

$$
\int \frac{d^{D-2} q}{(2 \pi)^{D-2}} e^{i \vec{q} \cdot \vec{b}}|\vec{q}|=-\frac{1}{2 \pi} \frac{1}{b^{3}}+\mathcal{O}(D-4),
$$

we obtain

$\left.\tilde{\mathcal{A}}_{\mathrm{FFR}}^{(1)}\left(1^{\phi}, 2^{\phi}, 3^{--}, 4^{++}\right)\right|_{\omega}=i Q^{2}\left(\frac{\kappa}{2}\right)^{2}\left(\frac{\alpha_{\gamma}}{4}\right) \frac{\omega}{256 \pi} \frac{1}{b^{3}}$,

$\left.\tilde{\mathcal{A}}_{\mathrm{FFR}}^{(1)}\left(1^{\phi}, 2^{\phi}, 3^{++}, 4^{++}\right)\right|_{\omega}=0$,

In this case the eikonal phase matrix is diagonal and the subleading contribution $\delta_{1, \mathrm{FFR}}$ is immediately seen to be

$$
\delta_{1, \mathrm{FFR}}=Q^{2}\left(\frac{\kappa}{2}\right)^{2}\left(\frac{\alpha_{\gamma}}{4}\right) \frac{\omega}{256 \pi} \frac{1}{b^{3}} \mathbb{1}_{2} .
$$

The new contribution to the graviton deflection angle due to the FFR interaction is then obtained using (2.16):

$\Delta \theta_{\mathrm{FFR}}=-Q^{2}\left(\frac{\kappa}{2}\right)^{2}\left(\frac{\alpha_{\gamma}}{4}\right) \frac{3}{256 \pi} \frac{1}{b^{4}}=-Q^{2} G\left(\frac{\alpha_{\gamma}}{4}\right) \frac{3}{32} \frac{1}{b^{4}}$.

Applying (2.17) to (4.54) we find the additional contribution to the time delay associated to the bending of a graviton in the FFR theory:

$\Delta t_{\mathrm{FFR}}=Q^{2}\left(\frac{\kappa}{2}\right)^{2}\left(\frac{\alpha_{\gamma}}{4}\right) \frac{1}{256 \pi} \frac{1}{b^{3}}=Q^{2} G\left(\frac{\alpha_{\gamma}}{4}\right) \frac{1}{32} \frac{1}{b^{3}}$.

The bending in this case is due to the electric charge $Q$ of the black hole, not to its mass, which does not appear in either (4.55) or (4.56). We conclude that in order to avoid possible causality violation due to time advance the coefficient of the FFR interaction must obey the positivity constraint

$$
\alpha_{\gamma}>0
$$

\section{E. Photon deflection angle and time delay in EH + FFR}

In this section we consider the photon deflection angle and the time delay/advance arising from the FFR interaction. Compared to the case of graviton bending considered in the previous section, there is a nonvanishing tree-level contribution to the deflection, thus we consider the leading and subleading eikonal cases separately.

\section{Leading eikonal}

The first contribution we consider arises from the EH tree-level amplitude (3.28), which in the eikonal approximation becomes ${ }^{10}$

$$
\mathcal{A}_{\mathrm{EH}}^{(0)}\left(1^{\phi}, 2^{\phi}, 3^{-}, 4^{+}\right) \simeq i\left(\frac{\kappa}{2}\right)^{2} \frac{(2 m \omega)^{2}}{\vec{q}^{2}},
$$

or, upon transforming to impact parameter,

$$
\tilde{\mathcal{A}}_{\mathrm{EH}}^{(0)}\left(1^{\phi}, 2^{\phi}, 3^{-}, 4^{+}\right) \simeq i\left(\frac{\kappa}{2}\right)^{2} m \omega f(-2, D-2) .
$$

Note that (4.58) has the same form as the two-scalar twograviton amplitude in the eikonal approximation, first equation in (4.1), as consequence of the equivalence principle.

At tree-level the helicity-preserving FFR amplitude (3.26) is purely a contact term, while the helicity-flip amplitude is given in (3.31). The leading contribution in the eikonal limit is then

$\mathcal{A}_{\mathrm{FFR}}^{(0)}\left(1^{\phi}, 2^{\phi}, 3^{-}, 4^{+}\right) \simeq 0$,

$\mathcal{A}_{\mathrm{FFR}}^{(0)}\left(1^{\phi}, 2^{\phi}, 3^{+}, 4^{+}\right) \simeq i\left(\frac{\kappa}{2}\right)^{2}\left(\frac{\alpha_{\gamma}}{4}\right)(2 m \omega)^{2} \frac{q^{2}}{|\vec{q}|^{2}}$,

where we used $[34]^{2}=-q^{2}$. Transforming the nonvanishing helicity-flip amplitude to impact parameter space we obtain

$\tilde{\mathcal{A}}_{\mathrm{FFR}}^{(0)}\left(1^{\phi}, 2^{\phi}, 3^{+}, 4^{+}\right) \simeq i\left(\frac{\kappa}{2}\right)^{2}\left(\frac{\alpha_{\gamma}}{4}\right) \frac{m \omega}{\overline{\mathfrak{b}}^{2}} \xi^{\prime \prime} f(-2, D-2)$,

where

\footnotetext{
${ }^{10} \mathrm{We}$ recall from Sec. III D 2 that $\mathcal{A}_{\mathrm{EH}}^{(0)}\left(1^{\phi}, 2^{\phi}, 3^{+}, 4^{+}\right)=$ $\mathcal{A}_{\mathrm{EH}}^{(0)}\left(1^{\phi}, 2^{\phi}, 3^{-}, 4^{-}\right)=0$.
} 


$$
\xi^{\prime \prime}=\left(\frac{D}{2}-2\right)\left(\frac{D}{2}-1\right)
$$

Defining

$$
\delta_{0}^{\gamma}=\delta_{0, \mathrm{EH}}^{\gamma}+\delta_{0, \mathrm{FFR}}^{\gamma}
$$

we can combine (4.59) and (4.61) into a single leading eikonal phase matrix ${ }^{11}$

$\delta_{0, \mathrm{FFR}}^{\gamma}=\left(\frac{\kappa}{2}\right)^{2} m \omega f(-2, D-2)\left(\begin{array}{cc}1 & \left(\frac{\alpha_{\gamma}}{4}\right) \frac{\xi^{\prime \prime}}{\overline{\mathfrak{b}}^{2}} \\ \left(\frac{\alpha_{\gamma}}{4}\right) \frac{\xi^{\prime \prime}}{\mathfrak{b}^{2}} & 1\end{array}\right)$,

which, upon expanding around $D=4$, reduces to

$$
\delta_{0, \mathrm{FFR}}^{\gamma}=-\left(\frac{\kappa}{2}\right)^{2} \frac{m \omega}{2 \pi}\left(\begin{array}{cc}
\frac{1}{4-D}+\log b & -\left(\frac{\alpha_{\gamma}}{4}\right) \frac{1}{2 \overline{\mathfrak{b}}^{2}} \\
-\left(\frac{\alpha_{\gamma}}{4}\right) \frac{1}{2 \mathfrak{b}^{2}} & \frac{1}{4-D}+\log b
\end{array}\right) .
$$

Next, in order to test the expected exponentiation property of the leading eikonal phase matrix, we consider the terms of $\mathcal{O}\left(\omega^{2}\right)$ in the one-loop amplitudes. These are given in impact parameter space by

$$
\begin{aligned}
& \left.\tilde{\mathcal{A}}_{\mathrm{EH}}^{(1)}\left(1^{\phi}, 2^{\phi}, 3^{-}, 4^{+}\right)\right|_{\omega^{2}} \\
& \quad=-\left(\frac{\kappa}{2}\right)^{4}(m \omega)^{2} \frac{f(D-6, D-2)}{2 \pi(D-4)}, \\
& \left.\tilde{\mathcal{A}}_{\mathrm{FFR}}^{(1)}\left(1^{\phi}, 2^{\phi}, 3^{+}, 4^{+}\right)\right|_{\omega^{2}} \\
& \quad=-\left(\frac{\kappa}{2}\right)^{4}\left(\frac{\alpha_{\gamma}}{4}\right) \frac{(m \omega)^{2}}{\overline{\mathfrak{b}}^{2}}(D-3) \frac{f(D-6, D-2)}{2 \pi},
\end{aligned}
$$

which are obtained from (3.29) and (3.32). In matrix form,

$$
\begin{aligned}
\tilde{\mathcal{A}}_{\omega^{2}}^{(1)}= & -\left(\frac{\kappa}{2}\right)^{2} \frac{(m \omega)^{2}}{2 \pi} f(D-6, D-2) \\
& \times\left(\begin{array}{cc}
\frac{1}{D-4} & \left(\frac{\alpha_{\gamma}}{4}\right) \frac{D-3}{\overline{\mathfrak{b}}^{2}} \\
\left(\frac{\alpha_{\gamma}}{4}\right) \frac{D-3}{\mathfrak{b}^{2}} & \frac{1}{D-4}
\end{array}\right) .
\end{aligned}
$$

Expanding around $D=4$ we find that $\tilde{\mathcal{A}}_{\omega^{2}}^{(1)}$ satisfies the matrix equation

\footnotetext{
${ }^{11}$ There is no need here to separate the $\mathrm{EH}$ and theFFRcontributions, since we consider only photon bending coming from this source.
}

$$
\tilde{\mathcal{A}}_{\omega^{2}}^{(1)}=-\frac{1}{2}\left(\delta_{0}\right)^{2}+\mathcal{O}(D-4),
$$

as expected.

\section{Subleading eikonal}

Next we consider the subleading eikonal phase. The only nonvanishing $\mathrm{EH}$ contribution comes from the one-loop massive triangles in the helicity-preserving amplitude (3.29), and reads

$$
\left.\tilde{\mathcal{A}}_{\mathrm{EH}}^{(1)}\left(1^{\phi}, 2^{\phi}, 3^{-}, 4^{+}\right)\right|_{\omega}=i\left(\frac{\kappa}{2}\right)^{4} \frac{15}{256 \pi} \frac{m^{2} \omega}{b} .
$$

Just as in the case of the leading eikonal phase, the bending angle of photons in pure $\mathrm{EH}$ comes is the same as the graviton bending (4.11) thanks to the equivalence principle.

The contributions coming from the FFR interaction are obtained from (3.33) and (3.32), and in impact parameter space are

$$
\begin{aligned}
& \left.\tilde{\mathcal{A}}_{\mathrm{FFR}}^{(1)}\left(1^{\phi}, 2^{\phi}, 3^{-}, 4^{+}\right)\right|_{\omega}=0 \\
& \left.\tilde{\mathcal{A}}_{\mathrm{FFR}}^{(1)}\left(1^{\phi}, 2^{\phi}, 3^{+}, 4^{+}\right)\right|_{\omega}=i\left(\frac{\kappa}{2}\right)^{4}\left(\frac{\alpha_{\gamma}}{4}\right) \frac{45}{1024 \pi} \frac{m^{2} \omega}{b} \frac{1}{\overline{\mathfrak{b}}^{2}} .
\end{aligned}
$$

Combining these results into a subleading eikonal phase matrix we get

$$
\delta_{1, \mathrm{FFR}}^{\gamma}=\left(\frac{\kappa}{2}\right)^{4} \frac{15}{256 \pi} \frac{m^{2} \omega}{b}\left(\begin{array}{cc}
1 & \left(\frac{\alpha_{\gamma}}{4}\right) \frac{3}{4 \overline{\mathfrak{b}}^{2}} \\
\left(\frac{\alpha_{\gamma}}{4}\right) \frac{3}{4 \mathfrak{b}^{2}} & 1
\end{array}\right) .
$$

\section{Deflection angle and time delay}

Having computed the eikonal phase matrix at leading and subleading order, we can now extract the light bending angle and time advance/delay. First we compute the eigenvalues of the leading eikonal phase matrix (4.65):

$\delta_{0, \mathrm{FFR}}^{\gamma(1,2)}=-\left(\frac{\kappa}{2}\right)^{2} \frac{m \omega}{2 \pi}\left[\left(\frac{1}{4-D}+\log b\right) \mp\left(\frac{\alpha_{\gamma}}{4}\right) \frac{2}{b^{2}}\right]$,

which match qualitatively the result of photon deflection in a shockwave background (see $[89,75]$ for related work), while at subleading order we have

$$
\delta_{0, \mathrm{FFR}}^{\gamma(1,2)}=\left(\frac{\kappa}{2}\right)^{4} \frac{15}{256 \pi} \frac{m^{2} \omega}{b}\left[1 \pm\left(\frac{\alpha_{\gamma}}{4}\right) \frac{3}{b^{2}}\right]
$$

Using once again (2.16), we find the light bending angle up to $\mathcal{O}\left(G^{2}\right)$ : 


$$
\begin{aligned}
\Delta \theta_{\mathrm{FFR}}^{\gamma(1,2)} & =-\left(\frac{\kappa}{2}\right)^{2} \frac{1}{2 \pi} \frac{m}{b}\left\{1 \pm\left(\frac{\alpha_{\gamma}}{4}\right) \frac{4}{b^{2}}+\left(\frac{\kappa}{2}\right)^{2} \frac{15}{128} \frac{m}{b}\left[1 \pm\left(\frac{\alpha_{\gamma}}{4}\right) \frac{9}{b^{2}}\right]\right\} \\
& =-\frac{4 G m}{b}\left\{1 \pm\left(\frac{\alpha_{\gamma}}{4}\right) \frac{4}{b^{2}}+\frac{15 \pi}{16} \frac{G m}{b}\left[1 \pm\left(\frac{\alpha_{\gamma}}{4}\right) \frac{9}{b^{2}}\right]\right\}
\end{aligned}
$$

Finally, applying (2.17) to (4.72) and (4.73) we arrive at our result for the time delay:

$$
\begin{aligned}
\Delta t_{\mathrm{FFR}}^{\gamma(1,2)} & =\left(\frac{\kappa}{2}\right)^{2} \frac{m}{2 \pi}\left\{\log \frac{b_{0}}{b} \pm\left(\frac{\alpha_{\gamma}}{4}\right) \frac{2}{b^{2}}+\left(\frac{\kappa}{2}\right)^{2} \frac{15}{128} \frac{m}{b}\left[1 \pm\left(\frac{\alpha_{\gamma}}{4}\right) \frac{3}{b^{2}}\right]\right\} \\
& =4 G m\left\{\log \frac{b_{0}}{b} \pm\left(\frac{\alpha_{\gamma}}{4}\right) \frac{2}{b^{2}}+\frac{15 \pi}{16} \frac{G m}{b}\left[1 \pm\left(\frac{\alpha_{\gamma}}{4}\right) \frac{3}{b^{2}}\right]\right\} .
\end{aligned}
$$

We note that the $\mathcal{O}\left(G \alpha_{\gamma}\right)$ part of our result (4.74) is in precise agreement with [73] while it disagrees with [72]. ${ }^{12}$ Note that (4.75) generically leads to a potential time advance and causality violation independent of the sign of the coupling $\alpha_{\gamma}$. This parallels the situation for the $R^{3}$ interaction which requires an appropriate UV completion to restore causality [87].

\section{ACKNOWLEDGMENTS}

We would like to thank Tim Clifton, Claudia de Rham, Lance Dixon, Gregory Korchemsky, David Kosower, Lorenzo Magnea, Rodolfo Russo, Chris White and Alexander Zhiboedov for interesting discussions and comments. We also thank the organizers of the Paris winter workshop "The Infrared in QFT", where our results were presented. This work was supported by the Science and Technology Facilities Council (STFC) Consolidated Grant No. ST/P000754/1 "String theory, gauge theory \& duality", and by the European Union's Horizon 2020 research and innovation programme under the Marie SkłodowskaCurie grant agreement No. 764850 "SAGEX”.

\section{APPENDIX A: RELEVANT INTEGRALS}

In this section we give the explicit expression for the integral functions appearing in our results. These expressions are expanded in $\epsilon$ up to the relevant orders, and only terms with an $s$-channel discontinuity are kept.

$$
\begin{gathered}
I_{2}(s) \simeq \frac{i}{16 \pi^{2}}\left[\frac{1}{\epsilon}-\log (-s)\right], \\
I_{3}(s) \simeq \frac{i}{16 \pi^{2} s}\left[\frac{1}{\epsilon^{2}}-\frac{\log (-s)}{\epsilon}+\frac{1}{2} \log ^{2}(-s)\right], \quad(\mathrm{A} 1) \\
I_{3}(s ; m) \simeq-\frac{i}{32}\left[\frac{1}{m \sqrt{-s}}+\frac{\log \left(-s / m^{2}\right)}{\pi^{2} m^{2}}\right], \quad(\mathrm{A} 3) \\
I_{4}(s, t ; m)+I_{4}(s, u ; m) \simeq-\frac{1}{8 \pi} \frac{1}{m \omega} \frac{1}{D-4}(-s)^{\frac{D-6}{2}} \\
\simeq-\frac{1}{16 \pi s(m \omega)}\left[\frac{1}{\epsilon}-\log \left(-\frac{s}{m^{2}}\right)\right] .
\end{gathered}
$$

\section{APPENDIX B: FEYNMAN RULES}

Below we list some of the Feynman rules used to obtain the new tree-level amplitudes quoted in the paper. Note that $1_{\phi_{m}}$ represents a massive scalar with momentum $p_{1}, 1^{\alpha}$ represents a photon with momentum $p_{1}$, and $3^{\mu \nu}$ represents a graviton with momentum $p_{3}$ :

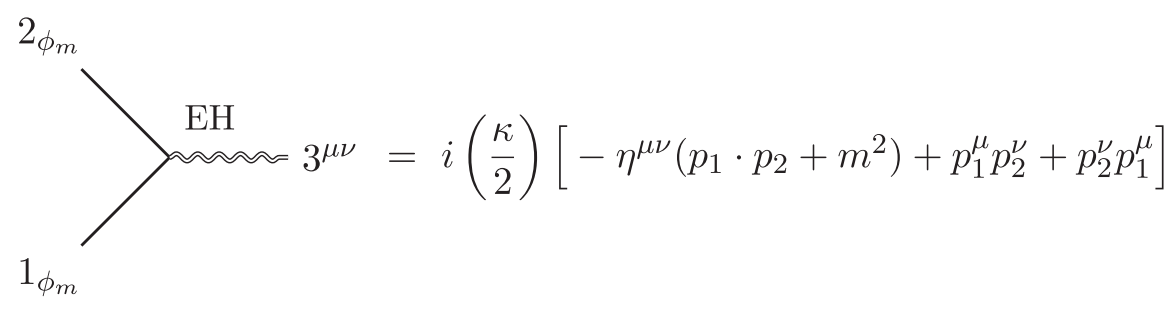

\footnotetext{
${ }^{12}$ The result of [72] for $\Delta \theta_{\mathrm{FFR}}^{\gamma}$ was already identified as incorrect in [73] due to an inappropriate definition of the deflection angle.
} 

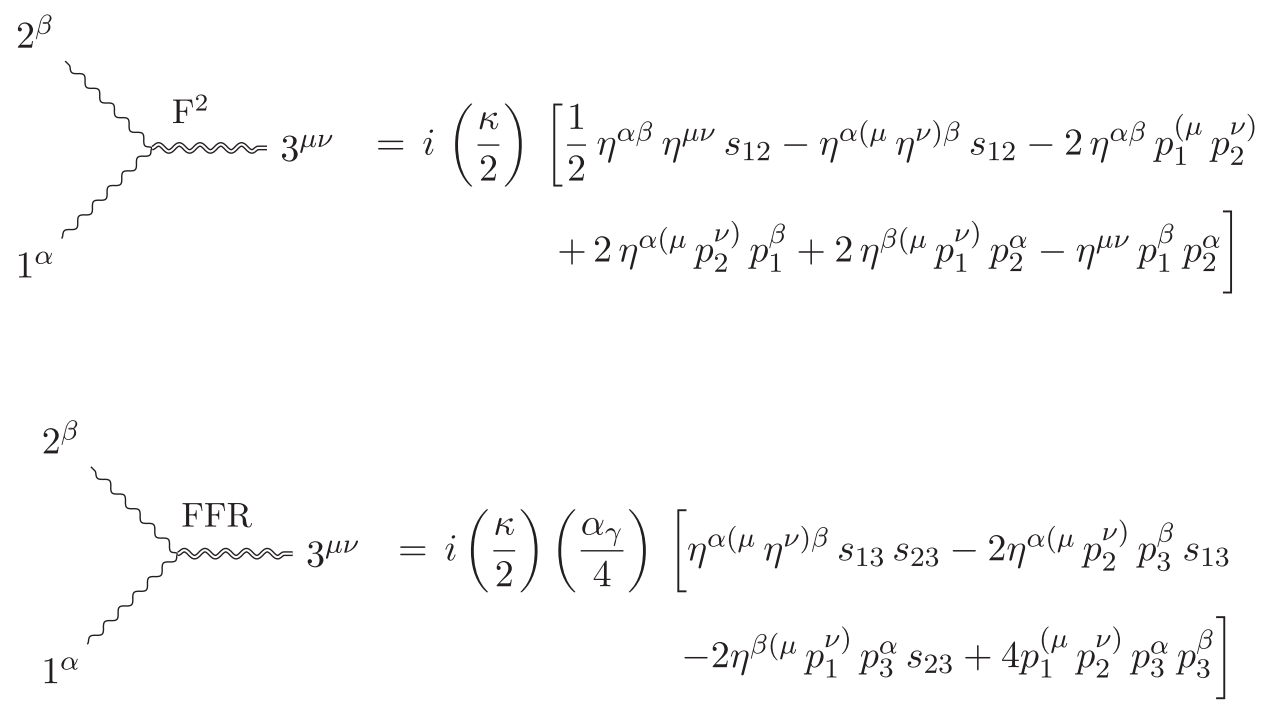

\section{APPENDIX C: THE TREE-LEVEL AMPLITUDES}

In this appendix we collect for the reader's convenience all the tree-level amplitudes we have used in our derivations. All are consistent with the normalizations of (1.1), also we assume all momenta to be outgoing.

$$
\begin{gathered}
\mathcal{A}_{\mathrm{SQED}}^{(0)}\left(1^{\phi}, 2^{\phi}, 3^{+}, 4^{+}\right)=e^{2} m^{2} \frac{[34]^{2}}{s}\left(\frac{i}{t-m^{2}}+\frac{i}{u-m^{2}}\right), \\
\mathcal{A}_{\mathrm{EH}}^{(0)}\left(1^{+}, 2^{-}, 3^{++}, 4^{--}\right)=-i\left(\frac{\kappa}{2}\right)^{2}[13]^{2}\langle 24\rangle^{2} \frac{\langle 4|1| 3]^{2}}{s t u}, \\
\mathcal{A}_{\mathrm{EH}}^{(0)}\left(1^{\phi}, 2^{\phi}, 3^{--}, 4^{++}\right)=-\left(\frac{\kappa}{2}\right)^{2} \frac{\langle 3|1| 4]^{4}}{s^{2}}\left[\frac{i}{t-m^{2}}+\frac{i}{u-m^{2}}\right], \\
\mathcal{A}_{\mathrm{EH}}^{(0)}\left(1^{++}, 2^{++}, 3^{--}, 4^{--}\right)=i\left(\frac{\kappa}{2}\right)^{2} \frac{s_{12^{2}} s_{13}}{s_{14}} \frac{\langle 34\rangle^{8}}{\langle 12\rangle^{4}\langle 23\rangle^{4}\langle 34\rangle^{4}\langle 41\rangle^{4}}, \\
\mathcal{A}_{\mathrm{EH}}^{(0)}\left(1^{\phi}, 2^{\phi}, 3^{++}, 4^{++}\right)=-\left(\frac{\kappa}{2}\right)^{2} m^{4} \frac{[34]^{2}}{\langle 34\rangle^{2}}\left[\frac{i}{t-m^{2}}+\frac{i}{u-m^{2}}\right], \\
\left.\mathcal{A}_{\mathrm{EH}}^{(0)}\left(1^{\phi}, 2^{\phi}, 3^{+}, 4^{+}\right)=0,4^{+}\right)=i\left(\frac{\kappa}{2}\right)^{2} \frac{\langle 3|1| 4]^{2}}{s}, \\
\mathcal{A}_{R^{4}}^{(0)}\left(1^{++}, 2^{++}, 3^{++}, 4^{++}\right)=i \beta\left(\frac{\kappa}{2}\right)^{2}\left([12]^{4}[34]^{4}+[13]^{4}[24]^{4}+[14]^{4}[23]^{4}\right), \\
\mathcal{A}_{R^{3}}^{(0)}\left(1^{\phi}, 2^{\phi}, 3^{++}, 4^{++}\right)=i\left(\frac{\kappa}{2}\right)^{2}\left(\frac{\alpha^{\prime}}{4}\right)^{2}[34]^{4} \frac{\left(t-m^{2}\right)\left(u-m^{2}\right)}{s}, \\
\mathcal{A}_{R^{3}}^{(0)}\left(1^{\phi}, 2^{\phi}, 3^{--}, 4^{++}\right)=0, \\
A_{R^{3}}\left(1^{++}, 2^{++}, 3^{++}, 4^{--}\right)=-i\left(\frac{\kappa}{2}\right)^{2}\left(\frac{\alpha^{\prime}}{4}\right)^{2}(\langle 41\rangle[13]\langle 34\rangle)^{2} \frac{[12][23][31]}{\langle 12\rangle\langle 23\rangle\langle 31\rangle},
\end{gathered}
$$




$$
\begin{aligned}
& \mathcal{A}_{R^{4}}^{(0)}\left(1^{++}, 2^{++}, 3^{--}, 4^{--}\right)=i \tilde{\beta}\left(\frac{\kappa}{2}\right)^{2}[12]^{4}\langle 34\rangle^{4} \\
& \mathcal{A}_{R^{4}}^{(0)}\left(1^{\phi}, 2^{\phi}, 3^{h_{3}}, 4^{h_{4}}\right)=0 \quad \text { with } \quad h_{3}, h_{4} \in\{+,-,++,--\}, \\
& \mathcal{A}_{\mathrm{FFR}}^{(0)}\left(1^{+}, 2^{+}, 3^{++}\right)=i\left(\frac{\kappa}{2}\right)\left(\frac{\alpha_{\gamma}}{4}\right)[13]^{2}[23]^{2}, \\
& \mathcal{A}_{\mathrm{FFR}}^{(0)}\left(1^{+}, 2^{+}, 3^{--}, 4^{++}\right)=-i\left(\frac{\kappa}{2}\right)^{2}\left(\frac{\alpha_{\gamma}}{4}\right)[12]^{2} \frac{\langle 3|1| 4]^{4}}{s t u}, \\
& \mathcal{A}_{\mathrm{FFR}}^{(0)}\left(1^{+}, 2^{+}, 3^{++}, 4^{++}\right)=i\left(\frac{\kappa}{2}\right)^{2}\left(\frac{\alpha_{\gamma}}{4}\right)\left(\frac{[13]^{2}[34]^{2}[42]^{2}}{s_{13}}+\frac{[23]^{2}[34]^{2}[41]^{2}}{s_{23}}\right), \\
& \mathcal{A}_{\mathrm{FFR}}^{(0)}\left(1^{\phi}, 2^{\phi}, 3^{-}, 4^{+}\right)=-i\left(\frac{\kappa}{2}\right)^{2}\left(\frac{\alpha_{\gamma}}{4}\right)\langle 3|1| 4]^{2}, \\
& \mathcal{A}_{\mathrm{FFR}}^{(0)}\left(1^{\phi}, 2^{\phi}, 3^{+}, 4^{+}\right)=-i\left(\frac{\kappa}{2}\right)^{2}\left(\frac{\alpha_{\gamma}}{4}\right)[34]^{2}\left[\frac{\left(t-m^{2}\right)\left(u-m^{2}\right)}{s}+m^{2}\right] .
\end{aligned}
$$

\section{APPENDIX D: THE FOUR-GRAVITON AMPLITUDES IN $\boldsymbol{R}^{4}$}

In this appendix we show how the most generic fourgraviton amplitude in an $R^{4}$ background can be constructed just from little-group considerations and dimensional analysis, without looking at any Lagrangian. We begin by noting that the coupling constant of the four-point amplitude has two powers of $\kappa([\kappa]=-1)$ and it is proportional to the coupling constant of the $R^{4}$ interaction $\beta([\beta]=-6)$. Furthermore, the nature of the new interaction implies that the four-point amplitude is just a contact term. Mass dimension and scaling under little-group transformations fix the form of the possible amplitudes completely:

$$
\begin{gathered}
\mathcal{A}_{R^{4}}^{(0)}\left(1^{++}, 2^{++}, 3^{++}, 4^{++}\right)=i \beta\left(\frac{\kappa}{2}\right)^{2} \tilde{\lambda}_{1}^{\otimes 4} \tilde{\lambda}_{2}^{\otimes 4} \tilde{\lambda}_{3}^{\otimes 4} \tilde{\lambda}_{4}^{\otimes 4}, \\
\mathcal{A}_{R^{4}}^{(0)}\left(1^{++}, 2^{++}, 3^{++}, 4^{--}\right)=0, \\
\mathcal{A}_{R^{4}}^{(0)}\left(1^{++}, 2^{++}, 3^{--}, 4^{--}\right)=i \tilde{\beta}\left(\frac{\kappa}{2}\right)^{2} \tilde{\lambda}_{1}^{\otimes 4} \tilde{\lambda}_{2}^{\otimes 4} \lambda_{3}^{\otimes 4} \lambda_{4}^{\otimes 4} .
\end{gathered}
$$

We can now introduce the convenient variables

$$
a:=[12][34], \quad b:=-[13][24], \quad c:=[14][23],
$$

in terms of which the all-plus amplitude can be written in such a way that permutation invariance is manifest. By saturating the spinor indices of (D1) with the LeviCivita tensor in all possible ways one gets four distinct combinations:

$$
\begin{aligned}
& \mathcal{A}_{R^{4}}^{(0)}\left(1^{++}, 2^{++}, 3^{++}, 4^{++}\right) \\
& =i \beta\left(\frac{\kappa}{2}\right)^{2}\left\{\begin{array}{l}
a^{4}+b^{4}+c^{4} \\
a^{2} b^{2}+a^{2} c^{2}+b^{2} c^{2} \\
a^{3} b+a b^{3}+a^{3} c+a c^{3}+b^{3} c+b c^{3} \\
a^{2} b c+a b^{2} c+a b c^{2}
\end{array}\right.
\end{aligned}
$$

However, using the Schouten identity, which in terms of these variables reads

$$
a+b+c=0
$$

one can show that there is actually only one independent combination, which we will take to be the first of (D5). We will then define the all-plus amplitude to be

$$
\begin{aligned}
& \mathcal{A}_{R^{4}}^{(0)}\left(1^{++}, 2^{++}, 3^{++}, 4^{++}\right) \\
& \quad=i \beta\left(\frac{\kappa}{2}\right)^{2}\left([12]^{4}[34]^{4}+[13]^{4}[24]^{4}+[14]^{4}[23]^{4}\right) .
\end{aligned}
$$

In the presence of a parity-invariant theory, the amplitude corresponding to (D7) with all helicities flipped is simply obtained by replacing $[j i] \rightarrow\langle i j\rangle$, otherwise it should be considered to have an independent normalization.

For the MHV amplitude (D3) there is only one possible structure, and we define the corresponding amplitude as

$$
\mathcal{A}_{R^{4}}^{(0)}\left(1^{++}, 2^{++}, 3^{--}, 4^{--}\right)=i \tilde{\beta}\left(\frac{\kappa}{2}\right)^{2}[12]^{4}\langle 34\rangle^{4}
$$

The derivation of these amplitudes from the Lagrangian (1.3) is presented in Sec. III C. 
[1] Y. Iwasaki, Fourth-order gravitational potential based on quantum field theory, Lett. Nuovo Cimento 1, 783 (1971).

[2] R. Feynman, Quantum theory of gravitation, Acta Phys. Pol. 24, 697 (1963).

[3] B. R. Holstein and J. F. Donoghue, Classical Physics and Quantum Loops, Phys. Rev. Lett. 93, 201602 (2004).

[4] N. E. J. Bjerrum-Bohr, J. F. Donoghue, and B. R. Holstein, Quantum gravitational corrections to the nonrelativistic scattering potential of two masses, Phys. Rev. D 67, 084033 (2003); Erratum, 71, 069903 (2005).

[5] J.F. Donoghue, General relativity as an effective field theory: The leading quantum corrections, Phys. Rev. D 50, 3874 (1994).

[6] Z. Bern, L. J. Dixon, D. C. Dunbar, and D. A. Kosower, One loop $n$-point gauge theory amplitudes, unitarity and collinear limits, Nucl. Phys. B425, 217 (1994).

[7] Z. Bern, L. J. Dixon, D. C. Dunbar, and D. A. Kosower, Fusing gauge theory tree amplitudes into loop amplitudes, Nucl. Phys. B435, 59 (1995).

[8] D. Neill and I. Z. Rothstein, Classical space-times from the S matrix, Nucl. Phys. B877, 177 (2013).

[9] N. E. J. Bjerrum-Bohr, J. F. Donoghue, and P. Vanhove, On-shell techniques and universal results in quantum gravity, J. High Energy Phys. 02 (2014) 111.

[10] N. E. J. Bjerrum-Bohr, J. F. Donoghue, B. R. Holstein, L. Plante, and P. Vanhove, Bending of Light in Quantum Gravity, Phys. Rev. Lett. 114, 061301 (2015).

[11] N. E. J. Bjerrum-Bohr, J. F. Donoghue, B. R. Holstein, L. Plante, and P. Vanhove, Light-like scattering in quantum gravity, J. High Energy Phys. 11 (2016) 117.

[12] D. Bai and Y. Huang, More on the bending of light in quantum gravity, Phys. Rev. D 95, 064045 (2017).

[13] H.-H. Chi, Graviton bending in quantum gravity from oneloop amplitudes, Phys. Rev. D 99, 126008 (2019).

[14] T. Damour and G. Schäfer, Lagrangians for point masses at the second post-Newtonian approximation of general relativity, Gen. Relativ. Gravit. 17, 879 (1985).

[15] J. B. Gilmore and A. Ross, Effective field theory calculation of second post-Newtonian binary dynamics, Phys. Rev. D 78, 124021 (2008).

[16] T. Damour, P. Jaranowski, and G. Schäfer, Dimensional regularization of the gravitational interaction of point masses, Phys. Lett. B 513, 147 (2001).

[17] L. Blanchet, T. Damour, and G. Esposito-Farese, Dimensional regularization of the third post-Newtonian dynamics of point particles in harmonic coordinates, Phys. Rev. D 69, 124007 (2004).

[18] Y. Itoh and T. Futamase, New derivation of a third postNewtonian equation of motion for relativistic compact binaries without ambiguity, Phys. Rev. D 68, 121501 (2003).

[19] S. Foffa and R. Sturani, Effective field theory calculation of conservative binary dynamics at third post-Newtonian order, Phys. Rev. D 84, 044031 (2011).

[20] P. Jaranowski and G. Schäfer, Towards the 4th postNewtonian Hamiltonian for two-point-mass systems, Phys. Rev. D 86, 061503 (2012).

[21] T. Damour, P. Jaranowski, and G. Schäfer, Nonlocal-intime action for the fourth post-Newtonian conservative dynamics of two-body systems, Phys. Rev. D 89, 064058 (2014).

[22] C. R. Galley, A. K. Leibovich, R. A. Porto, and A. Ross, Tail effect in gravitational radiation reaction: Time nonlocality and renormalization group evolution, Phys. Rev. D 93, 124010 (2016).

[23] T. Damour, P. Jaranowski, and G. Schäfer, Fourth postNewtonian effective one-body dynamics, Phys. Rev. D 91, 084024 (2015).

[24] T. Damour, P. Jaranowski, and G. Schäfer, Conservative dynamics of two-body systems at the fourth postNewtonian approximation of general relativity, Phys. Rev. D 93, 084014 (2016).

[25] L. Bernard, L. Blanchet, A. Bohé, G. Faye, and S. Marsat, Fokker action of nonspinning compact binaries at the fourth post-Newtonian approximation, Phys. Rev. D 93, 084037 (2016).

[26] L. Bernard, L. Blanchet, A. Bohé, G. Faye, and S. Marsat, Energy and periastron advance of compact binaries on circular orbits at the fourth post-Newtonian order, Phys. Rev. D 95, 044026 (2017).

[27] S. Foffa and R. Sturani, Dynamics of the gravitational twobody problem at fourth post-Newtonian order and at quadratic order in the Newton constant, Phys. Rev. D 87, 064011 (2013).

[28] S. Foffa, P. Mastrolia, R. Sturani, and C. Sturm, Effective field theory approach to the gravitational two-body dynamics, at fourth post-Newtonian order and quintic in the Newton constant, Phys. Rev. D 95, 104009 (2017).

[29] R. A. Porto and I. Z. Rothstein, Apparent ambiguities in the post-Newtonian expansion for binary systems, Phys. Rev. D 96, 024062 (2017).

[30] R. A. Porto, Lamb shift and the gravitational binding energy for binary black holes, Phys. Rev. D 96, 024063 (2017).

[31] S. Foffa, R. A. Porto, I. Rothstein, and R. Sturani, Conservative dynamics of binary systems to fourth postNewtonian order in the EFT approach II: Renormalized Lagrangian, Phys. Rev. D 100, 024048 (2019).

[32] J. Bluemlein, A. Maier, P. Marquard, and G. Schaefer, Fourth post-Newtonian Hamiltonian dynamics of twobody systems from an effective field theory approach, Nucl. Phys. B955, 115041 (2020).

[33] S. Foffa, P. Mastrolia, R. Sturani, C. Sturm, and W. J. T. Bobadilla, Static Two-Body Potential at Fifth PostNewtonian Order, Phys. Rev. Lett. 122, 241605 (2019).

[34] J. Bluemlein, A. Maier, and P. Marquard, Five-loop static contribution to the gravitational interaction potential of two point masses, Phys. Lett. B 800, 135100 (2020).

[35] J. Bluemlein, A. Maier, P. Marquard, and G. Schaefer, Testing binary dynamics in gravity at the sixth postNewtonian level, Phys. Lett. B 807, 135496 (2020).

[36] A. Einstein, L. Infeld, and B. Hoffmann, The gravitational equations and the problem of motion, Ann. Math. 39, 65 (1938).

[37] S. Foffa and R. Sturani, Effective field theory methods to model compact binaries, Classical Quantum Gravity 31, 043001 (2014). 
[38] I. Z. Rothstein, Progress in effective field theory approach to the binary inspiral problem, Gen. Relativ. Gravit. 46, 1726 (2014).

[39] R. A. Porto, The effective field theorist's approach to gravitational dynamics, Phys. Rep. 633, 1 (2016).

[40] M. Levi, Effective field theories of post-Newtonian gravity: A comprehensive review, Rep. Prog. Phys. 83, 075901 (2020).

[41] Z. Bern, C. Cheung, R. Roiban, C.-H. Shen, M. P. Solon, and M. Zeng, Scattering Amplitudes and the Conservative Hamiltonian for Binary Systems at Third Post-Minkowskian Order, Phys. Rev. Lett. 122, 201603 (2019).

[42] Z. Bern, C. Cheung, R. Roiban, C.-H. Shen, M. P. Solon, and M. Zeng, Black hole binary dynamics from the double copy and effective theory, J. High Energy Phys. 10 (2019) 206.

[43] C. Cheung and M. P. Solon, Classical gravitational scattering at $\mathcal{O}\left(G^{3}\right)$ from Feynman diagrams, J. High Energy Phys. 06 (2020) 144.

[44] A. Buonanno and T. Damour, Effective one-body approach to general relativistic two-body dynamics, Phys. Rev. D 59, 084006 (1999).

[45] T. Damour, Gravitational scattering, post-Minkowskian approximation and effective one-body theory, Phys. Rev. D 94, 104015 (2016).

[46] T. Damour, High-energy gravitational scattering and the general relativistic two-body problem, Phys. Rev. D 97, 044038 (2018).

[47] A. Luna, R. Monteiro, I. Nicholson, D. O'Connell, and C. D. White, The double copy: Bremsstrahlung and accelerating black holes, J. High Energy Phys. 06 (2016) 023.

[48] F. Cachazo and A. Guevara, Leading singularities and classical gravitational scattering, J. High Energy Phys. 02 (2020) 181.

[49] N. E. J. Bjerrum-Bohr, P. H. Damgaard, G. Festuccia, L. Plante, and P. Vanhove, General Relativity from Scattering Amplitudes, Phys. Rev. Lett. 121, 171601 (2018).

[50] J. Plefka, J. Steinhoff, and W. Wormsbecher, Effective action of dilaton gravity as the classical double copy of Yang-Mills theory, Phys. Rev. D 99, 024021 (2019).

[51] C. Cheung, I.Z. Rothstein, and M. P. Solon, From Scattering Amplitudes to Classical Potentials in the Post-Minkowskian Expansion, Phys. Rev. Lett. 121, 251101 (2018).

[52] D. A. Kosower, B. Maybee, and D. O'Connell, Amplitudes, observables, and classical scattering, J. High Energy Phys. 02 (2019) 137.

[53] A. Guevara, A. Ochirov, and J. Vines, Scattering of spinning black holes from exponentiated soft factors, J. High Energy Phys. 09 (2019) 056.

[54] Y. F. Bautista and A. Guevara, From scattering amplitudes to classical physics: Universality, double copy and soft theorems, arXiv:1903.12419.

[55] A. Koemans Collado, P. Di Vecchia, and R. Russo, Revisiting the 2PM eikonal and the dynamics of binary black holes, Phys. Rev. D 100, 066028 (2019).

[56] N. E. J. Bjerrum-Bohr, A. Cristofoli, and P. H. Damgaard, Post-Minkowskian scattering angle in Einstein gravity, arXiv:1910.09366.
[57] G. Kalin and R. A. Porto, From boundary data to bound states, J. High Energy Phys. 01 (2020) 072.

[58] S. Endlich, V. Gorbenko, J. Huang, and L. Senatore, An effective formalism for testing extensions to general relativity with gravitational waves, J. High Energy Phys. 09 (2017) 122.

[59] N. Sennett, R. Brito, A. Buonanno, V. Gorbenko, and L. Senatore, Gravitational-wave constraints on an effectivefield-theory extension of general relativity, arXiv: 1912.09917 [Phys. Rev. D (to be published)].

[60] A. Brandhuber and G. Travaglini, On higher-derivative effects on the gravitational potential and particle bending, J. High Energy Phys. 01 (2020) 010.

[61] W. T. Emond and N. Moynihan, Scattering amplitudes, black holes and leading singularities in cubic theories of gravity, J. High Energy Phys. 12 (2019) 019.

[62] P. van Nieuwenhuizen and C. C. Wu, On integral relations for invariants constructed from three riemann tensors and their applications in quantum gravity, J. Math. Phys. (N.Y.) 18, 182 (1977).

[63] J. Broedel and L. J. Dixon, Color-kinematics duality and double-copy construction for amplitudes from higher-dimension operators, J. High Energy Phys. 10 (2012) 091.

[64] M. H. Goroff and A. Sagnotti, Quantum gravity at two loops, Phys. Lett. 160B, 81 (1985).

[65] S. Fulling, R. C. King, B. Wybourne, and C. Cummins, Normal forms for tensor polynomials. 1: The Riemann tensor, Classical Quantum Gravity 9, 1151 (1992).

[66] A. A. Tseytlin, Ambiguity in the effective action in string theories, Phys. Lett. B 176, 92 (1986).

[67] R. R. Metsaev and A. A. Tseytlin, Order $\alpha^{\prime}$ (two loop) equivalence of the string equations of motion and the Sigma model Weyl invariance conditions: Dependence on the dilaton and the antisymmetric tensor, Nucl. Phys. B293, 385 (1987).

[68] M. Accettulli Huber, A. Brandhuber, S. De Angelis, and G. Travaglini, Note on the absence of $R^{2}$ corrections to Newton's potential, Phys. Rev. D 101, 046011 (2020).

[69] D. C. Dunbar, J. H. Godwin, G. R. Jehu, and W. B. Perkins, Loop amplitudes in an extended gravity theory, Phys. Lett. B 780, 41 (2018).

[70] M. Ruhdorfer, J. Serra, and A. Weiler, Effective field theory of gravity to all orders, J. High Energy Phys. 05 (2020) 083.

[71] D. J. Gross and E. Witten, Superstring modifications of Einstein's equations, Nucl. Phys. B277, 1 (1986).

[72] F. A. Berends and R. Gastmans, Quantum electrodynamical corrections to graviton-matter vertices, Ann. Phys. (N.Y.) 98, 225 (1976).

[73] I. Drummond and S. Hathrell, QED vacuum polarization in a background gravitational field and its effect on the velocity of photons, Phys. Rev. D 22, 343 (1980).

[74] T. J. Hollowood and G. M. Shore, The refractive index of curved spacetime: The fate of causality in QED, Nucl. Phys. B795, 138 (2008).

[75] G. Goon and K. Hinterbichler, Superluminality, black holes and EFT, J. High Energy Phys. 02 (2017) 134.

[76] I. I. Shapiro, Fourth Test of General Relativity, Phys. Rev. Lett. 13, 789 (1964). 
[77] H. Cheng and T. T. Wu, High-Energy Elastic Scattering in Quantum Electrodynamics, Phys. Rev. Lett. 22, 666 (1969).

[78] M. Lévy and J. Sucher, Eikonal approximation in quantum field theory, Phys. Rev. 186, 1656 (1969).

[79] H. D. Abarbanel and C. Itzykson, Relativistic Eikonal Expansion, Phys. Rev. Lett. 23, 53 (1969).

[80] D. Amati, M. Ciafaloni, and G. Veneziano, Superstring collisions at Planckian energies, Phys. Lett. B 197, 81 (1987).

[81] D. Amati, M. Ciafaloni, and G. Veneziano, Higher order gravitational deflection and soft Bremsstrahlung in Planckian energy superstring collisions, Nucl. Phys. B347, 550 (1990).

[82] D. N. Kabat and M. Ortiz, Eikonal quantum gravity and Planckian scattering, Nucl. Phys. B388, 570 (1992).

[83] R. Akhoury, R. Saotome, and G. Sterman, High energy scattering in perturbative quantum gravity at next to leading power, arXiv:1308.5204.

[84] Z. Bern, H. Ita, J. Parra-Martinez, and M. S. Ruf, Universality in the Classical Limit of Massless Gravitational Scattering, Phys. Rev. Lett. 125, 031601 (2020).

[85] Z. Bern, A. Luna, R. Roiban, C.-H. Shen, and M. Zeng, Spinning black hole binary dynamics, scattering amplitudes and effective field theory, arXiv:2005.03071.

[86] J. Parra-Martinez, M. S. Ruf, and M. Zeng, Extremal black hole scattering at $O\left(G^{3}\right)$ : Graviton dominance, eikonal exponentiation, and differential equations, arXiv: 2005.04236.

[87] X. O. Camanho, J. D. Edelstein, J. Maldacena, and A. Zhiboedov, Causality constraints on corrections to the graviton three-point coupling, J. High Energy Phys. 02 (2016) 020.

[88] T. J. Hollowood and G. M. Shore, Causality and microcausality in curved spacetime, Phys. Lett. B 655, 67 (2007).

[89] T. J. Hollowood and G. M. Shore, Causality violation, gravitational shockwaves and UV completion, J. High Energy Phys. 03 (2016) 129.

[90] K. Benakli, S. Chapman, L. Darmé, and Y. Oz, Superluminal graviton propagation, Phys. Rev. D 94, 084026 (2016).

[91] T. J. Hollowood and G. M. Shore, Causality, renormalizability and ultra-high energy gravitational scattering, J. Phys. A 49, 215401 (2016).

[92] C. de Rham and A. J. Tolley, Speed of gravity, Phys. Rev. D 101, 063518 (2020).

[93] C. de Rham, J. Francfort, and J. Zhang, Black hole gravitational waves in the effective field theory of gravity, Phys. Rev. D 102, 024079 (2020).

[94] D. Amati, M. Ciafaloni, and G. Veneziano, Classical and quantum gravity effects from Planckian energy superstring collisions, Int. J. Mod. Phys. A 03, 1615 (1988).

[95] G. D’Appollonio, P. Vecchia, R. Russo, and G. Veneziano, Microscopic unitary description of tidal excitations in high-energy string-brane collisions, J. High Energy Phys. 126 (2013) 11.

[96] A. Gruzinov and M. Kleban, Causality constrains higher curvature corrections to gravity, Classical Quantum Gravity 24, 3521 (2007).

[97] B. Bellazzini, C. Cheung, and G. N. Remmen, Quantum gravity constraints from unitarity and analyticity, Phys. Rev. D 93, 064076 (2016).

[98] I. B. Khriplovich and G. G. Kirilin, Zh. Eksp. Teor. Fiz. 125, 1219 (2004) [Quantum long range interactions in general relativity, J. Exp. Theor. Phys. 98, 1063 (2004)].

[99] Y. Iwasaki, Quantum theory of gravitation vs classical theory-fourth-order potential, Prog. Theor. Phys. 46, 1587 (1971).

[100] P. Di Vecchia, A. Luna, S. G. Naculich, R. Russo, G. Veneziano, and C. D. White, A tale of two exponentiations in $\mathcal{N}=8$ supergravity, Phys. Lett. B 798, 134927 (2019).

[101] P. Di Vecchia, S. G. Naculich, R. Russo, G. Veneziano, and C. D. White, A tale of two exponentiations in $\mathcal{N}=8$ supergravity at subleading level, J. High Energy Phys. 03 (2020) 173.

[102] N. E. J. Bjerrum-Bohr, B. R. Holstein, J. F. Donoghue, L. Plante, and P. Vanhove, Illuminating light bending, Proc. Sci., CORFU2016 (2017) 077 [arXiv:1704.01624].

[103] L. Eisenbud, The formal properties of nuclear collisions. Ph.D. thesis, Princeton University, 1948.

[104] D. Bohm, Quantum Theory (Prentice Hall, New York, 1951).

[105] E. P. Wigner, Lower limit for the energy derivative of the scattering phase shift, Phys. Rev. 98, 145 (1955).

[106] A. Guevara, Holomorphic classical limit for spin effects in gravitational and electromagnetic scattering, J. High Energy Phys. 04 (2019) 033.

[107] D. Nandan, J. Plefka, and G. Travaglini, All rational oneloop Einstein-Yang-Mills amplitudes at four points, J. High Energy Phys. 09 (2018) 011.

[108] R. Britto, F. Cachazo, B. Feng, and E. Witten, Direct Proof of Tree-Level Recursion Relation in Yang-Mills Theory, Phys. Rev. Lett. 94, 181602 (2005).

[109] T. Cohen, H. Elvang, and M. Kiermaier, On-shell constructibility of tree amplitudes in general field theories, J. High Energy Phys. 04 (2011) 053.

[110] A. Einstein, On the influence of gravitation on the propagation of light, Ann. Phys. (N.Y.) 35, 898 (1911).

[111] M. Ciafaloni and D. Colferai, Rescattering corrections and self-consistent metric in Planckian scattering, J. High Energy Phys. 10 (2014) 085. 